\title{
La estructura social de la industria biotecnológica en Francia: un estudio de las relaciones inter- organizacionales a nivel inter-individual
}

\author{
Alvaro Piña-Stranger ${ }^{1}$ - IRISSO (CNRS - Université Paris Dauphine) ${ }^{2}$
}

\begin{abstract}
Resumen
Diversos autores han mostrado la importancia de las relaciones de colaboración inter-organizacionales para el desempeño, las formas de gestión o la trayectoria de las empresas biotecnológicas. La mayoría de estos trabajos analizan exclusivamente los acuerdos contractuales entre las empresas y sus principales interlocutores. Este enfoque exclusivamente económico presenta una limitación mayor: la naturaleza de las relaciones contractuales no permite estudiar en detalle la manera en la que los actores cooperan. Nuestro estudio propone ampliar el análisis de estas relaciones inter-organizacionales al nivel inter-individual. Un estudio empírico sobre los dirigentes de la industria biotecnológica en el sector de la salud humana en Francia nos ha permitido cartografiar las relaciones que mantienen y los recursos que intercambian entre ellos. Nuestros resultados confirman la existencia de un sistema de intercambio denso y múltiple. Éste presenta una distribución jerárquica de los diversos tipos de recursos, en la que el centro se diferencia de la periferia por la presencia de relaciones más densas, múltiples y recíprocas. Sin embargo, el análisis comparado de las diferentes redes revela que las relaciones de consejo son bastante centralizadas, mientras que las relaciones de amistad siguen una distribución más homogénea. Sugerimos que este fenómeno forma parte de un mecanismo de compensación que permite a los actores menos centrales mantener relaciones inter-organizacionales. Por último, el análisis de los comportamientos relacionales nos ha permitido descubrir dos normas que orientan los intercambios. Estas normas reflejan en parte el difícil proceso de ajustamiento que debe atravesar un proyecto científico para salir del universo de la investigación académica y desarrollarse en una estructura privada: la empresa biotecnológica.
\end{abstract}

Palabras claves: industria biotecnológica - análisis de redes sociales cooperación entre competidores - relaciones inter-organizacionales

\begin{abstract}
Various authors have shown the importance of collaborative relationships for interorganizational performance, the mode of governance or the trajectory of biotechnology companies. Most of these works analyze the exclusive contractual agreements between companies and their main relationships among individuals. We show that this purely economic approach presents a major limitation: the nature of contractual relationships does not explore in detail how players cooperate. We propose to extend the study of these inter-organizational social relations, seen through the resource exchange in inter-individual. An empirical study on the leaders of the biotechnology industry in the area of human health in France has allowed us to map their relationships and resources they exchange them. Our results confirm the existence of a system of exchange dense and multiple. It presents a hierarchical distribution of various types of resources, where the centre

\footnotetext{
${ }^{1}$ Enviar correspondencia a: alvaro.pina-stranger@dauphine.fr

2 Agradezco el apoyo de la Ville de Paris que hizo este estudio posible y la ayuda de los actores que tuvieron la paciencia y la generosidad de responder a mis preguntas. Agradezco también a la asociación profesional France Biotech por su importante apoyo. Agradezco igualmente a los miembros del grupo ORIO (http://orio.dauphine.fr/) por sus consejos y comentarios.
} 
is different from the periphery relations denser, more numerous and more reciprocal. However, comparative analysis of different networks reveals that the relationships of the board are highly centralized, while those of friendship following a more even distribution. We suggest that this phenomenon is part of a compensation mechanism to less central actors to maintain inter-organizational relationships. Finally, two standards of the cooperation process, revealed by the relational behaviour of actors, have been discovered. We suggest that they reflect in part the difficult process of adjustment that must cross a science project out of the realm of academic research and develop in a private structure: the biotech company.

Keywords: Biotech industry - Social Network Analysis - cooperation - interorganizational relationships.

\section{I ntroducción}

La industria biotecnológica encarna intereses sociales y económicos mayores. En el área de la salud humana ${ }^{3}$, los nuevos conocimientos originarios de las ciencias biológicas han modificado desde hace ya 30 años la industria de los productos terapéuticos y la vida de millones de pacientes implicados por sus innovaciones. El descubrimiento de nuevos medicamentos depende en gran parte de las miles de pequeñas y medianas empresas biotecnológicas repartidas por el mundo. A medio camino entre la investigación académica (IA) y las grandes empresas farmacéuticas (Big-Pharmas), las empresas biotecnológicas juegan un rol motor en la transferencia de los conocimientos científicos hacia otros dominios de la vida social. Ellas tienen, en particular, una importancia creciente en los sectores sanitarios, académicos, políticos, industriales y financieros. Estas empresas innovadoras, que presenten un gran potencial de crecimiento, reciben enormes inversiones públicas y privadas. Los países desarrollados luchan por mejorar las condiciones económicas y científicas que les permitan acoger a estas empresas.

Aun así, el desarrollo de un nuevo medicamento es una apuesta arriesgada y los proyectos científicos que logran encontrar financiamiento son pocos. De estos, la gran mayoría no logrará introducir un producto en el mercado. Los empresarios científicos que deciden crear una empresa tienen la difícil misión de "transferir" un descubrimiento científico del mundo de la IA hacia el sector privado con el objetivo de desarrollar sus potencialidades terapéuticas. Si todo sale bien, pasarán gran parte de sus carreras en esto, pues el tiempo necesario para el desarrollo de un medicamento no es inferior a diez años.

${ }^{3}$ Hablaremos de industria biotecnología, de empresa de Biotech o de Biotech para indicar las empresas biotecnológicas que trabajan en el sector de la salud humana, sin especificar cada vez que no se trata de empresas biotecnológicas que trabajan en algún otro sector. 
El primer objetivo-obstáculo que el empresario debe superar es el de la propiedad intelectual. En Francia, muchos laboratorios están bajo la tutela de varios organismos públicos. Estos últimos son copropietarios de las patentes inscritas por sus científicos. El empresario debe negociar con esos organismos y promover un acuerdo entre ellos para obtener las licencias que le permitan "asegurar" financieramente el trabajo de investigación y desarrollo (ID). El segundo objetivoobstáculo atañe a los recursos financieros que este trabajo necesita. Las patentes y el equipo científico son a menudo los únicos activos con los que cuentan los empresarios al momento de presentar un proyecto a los inversionistas en capitalriesgo (CR). Si estos últimos entran en el capital de la empresa, aportarán los recursos necesarios al desarrollo de los proyectos, y, en contraparte, se convertirán en copropietarios de la empresa. En general 3 ó 4 recaudaciones de fondos (fund raising) son necesarias antes de poder introducir una empresa en el mercado público. En el intertanto, las empresas pueden firmar contratos de colaboración con alguna Big-Pharma. Típicamente, éstas procuran el dinero y la experiencia necesarios en las últimas fases de desarrollo. Por último, esta trayectoria no es posible sin las autorizaciones sucesivas de las agencias sanitarias que regulan los productos de salud. La multiplicidad de riesgos, y las expectativas sociales depositadas en los productos en desarrollo, hacen de esta industria una de las más exigentes y prometedoras de nuestros días.

El sector biotecnológico suscita un gran interés en los economistas y, en menor medida, en los sociólogos. Diversos autores han señalado la importancia de las colaboraciones inter-organizacionales para el desarrollo de las empresas. Sus trabajos cartografían las relaciones que mantienen las empresas biotecnológicas entre ellas y con sus principales interlocutores. El "perfil de colaboración" es examinado con el objetivo de establecer un vínculo con el desempeño económico, las formas de gestión o las trayectorias de las empresas. Estos estudios comparten una misma tesis general: el riesgo omnipresente y el alto nivel de exigencias científicas, industriales, de regulación y financieras que caracterizan esta industria, hacen que ningún actor sea capaz de poseer de manera individual todos los recursos necesarios al desarrollo de un producto terapéutico (ver por ejemplo Pisano, 1990, Powell, 1996, Oresingo y al., 2000). Estas empresas se verían, entonces, "obligadas" a intercambiar recursos entre ellas para aumentar sus probabilidades de éxito, incluso si, al mismo tiempo, deben luchar por acceder a las subvenciones públicas, los cupos en las incubadoras de empresas, las inversiones privadas o los contratos con las Big-Pharmas. En este sentido, los trabajos sobre la estructura y el funcionamiento de la industria biotecnológica se inscriben de manera 
general en la problemática de la cooperación entre competidores (White, 1981, Lazega, 2008).

La gran mayoría de estos trabajos reconstituyen las relaciones interorganizacionales examinando los contratos de las empresas. Este enfoque exclusivamente económico presenta una limitación mayor: la naturaleza de las relaciones contractuales no permite estudiar en detalle la manera en la que los actores cooperan. Los mecanismos de solidaridad, la construcción de los status o las estrategias relacionales, por ejemplo, permanecen invisibles en el análisis de los contratos. Sin embargo, la necesidad de observar las relaciones sociales a nivel inter-individual ha sido puesta en evidencia por varios trabajos sobre este sector (ver por ejemplo Lemarié y al., 2001, Powell y al., 2002, Owen-Smith y al., 2004, Casper, 2007). Estos autores ven en las relaciones contractuales un indicador de las relaciones sociales, aun cuando estas últimas no son observadas directamente. En un contexto en el que los empresarios tratan cada vez más de encontrarse en juntas, grupos de trabajo o de interés (Lazega y Mounier, 2002), una parte importante de los intercambios que mantienen los actores de esta industria se ve excluida de los análisis si se toman únicamente en cuenta los contratos, sin contar además con que muchos de estos contratos son a menudo mantenidos bajo confidencialidad. La infravaloración de las relaciones entre empresarios impide el desarrollo de análisis más precisos sobre la manera en la que se construye y funciona en parte la eficacidad de una industria. La estructura social de oportunidades y de limitaciones que se configura a través de las relaciones sociales escapa a la mayoría de los trabajos sobre este sector ya que a partir del momento en que las relaciones inter-individuales son supuestas, -y no observadas-, es muy difícil reconstituir las formas y lógicas sociales que participan al funcionamiento del colectivo. Sin embargo, no hemos encontrado aún una investigación que se interese al estudio de las relaciones inter-individuales que mantienen los actores en la industria biotecnológica ${ }^{4}$.

La investigación que presentamos en este artículo pretende colmar este vacío empírico y teórico. Defendemos la idea de que la estructura económica de este sector puede ser estudiada con un enfoque contractual, pero el estudio de la estructura social necesita el examen de las relaciones personales que mantienen los actores. Proponemos ampliar el estudio de las relaciones inter-organizacionales a las relaciones sociales, observadas a través del intercambio de recursos a nivel inter-individual. Estos recursos son difíciles de encontrar pues conciernen saberes

${ }^{4}$ Como indica Lazega (2008), estos estudios "son muy escasos debido a que los datos de este tipo son delicados, estratégicos y difíciles de obtener". 
nuevos, experiencias recientes, no estabilizadas (J affe y al., 1993). En la industria biotecnológica, las innovaciones científicas, organizacionales y económicas implicadas en Ia ID, impiden en gran parte la rutinización de las actividades. Cada equipo de dirección debe encontrar las soluciones ad hoc a los problemas que emergen en los proyectos de la empresa. En ese contexto, la adecuación de los recursos que circulan entre los dirigentes de las diferentes empresas es una cuestión decisiva y problemática. Son a menudo las relaciones con los competidores que dan acceso a las informaciones pertinentes. El estudio que presentamos tiene por objetivo examinar la manera en la que los dirigentes cooperan y se coordinan para evaluar y hacer accesibles estos recursos. Veremos en particular la estructura que caracteriza el sistema de intercambios, y las normas sociales que orientan su funcionamiento.

En la próxima sección revisaremos los aportes y las limitaciones del enfoque contractual en el estudio de las relaciones inter-organizacionales. Presentaremos a continuación un enfoque complementario, fundado en el análisis de las relaciones a nivel inter-individual, y las hipótesis que este enfoque nos permitirá explorar. En la sección 3 presentaremos la investigación que hemos diseñado para probar nuestras hipótesis, y en la sección 4 expondremos y discutiremos nuestros resultados.

\section{Relaciones inter-organizacionales en la industria biotecnológica}

Una buena parte de los trabajos sobre la industria biotecnológica se ha centrado en el análisis de las relaciones inter-organizacionales. En esta sección, presentaremos primero algunos de los principales resultados obtenidos por estos trabajos. En seguida, comentaremos los límites del enfoque exclusivamente contractual utilizado en la mayoría de los estudios. Por último, presentaremos un enfoque complementario para el análisis de las relaciones inter-organizacionales. Éste se caracteriza por el examen de las relaciones sociales, personalizadas, que permiten estudiar los mecanismos sociales que estructuran este sector industrial.

\subsection{Colaboraciones inter-organizacionales y formas de coordinación}

Diversos trabajos se han interesado en las relaciones inter-organizacionales en la industria biotecnológica en el área de la economía y, en menor medida, en el de la sociología económica. Estos trabajos se pueden dividir en tres subgrupos.

En el primero, se encuentran los autores que estudian les relaciones de colaboración en la industria biotecnológica. Estos analizan cómo la posición de las empresas en la estructura de relaciones determina su capacidad a desarrollarse, 
innovar o aprender (ver por ejemplo Powell y al., 1999, Senker y al., 1997, Baum y al., 2000, Zuker y al., 2001, Filiou, 2005, Salman y al., 2005).

Uno de los principales representantes de este primer grupo es Walter W. Powell. Él y sus colaboradores han establecido un cierto número de características de la industria biotecnológica. Su primer artículo importante examinaba las relaciones contractuales entre 230 empresas biotecnológicas y más de 1800 de sus colaboradores en un período de 8 años (1988-1995). Powell (1996) se interesa en un principio a los factores que promueven las relaciones de colaboración interorganizacionales. El autor muestra que ni el comportamiento puramente oportunista ni la necesidad de ir a buscar competencias complementarias explican el establecimiento de colaboraciones entre las empresas. Para Powell, el crecimiento del número de colaboración entre las empresas corresponde al desarrollo de un nuevo modo de coordinación entre los actores. Las colaboraciones serían el "lugar de la innovación" ya que ellas permiten acceder a nuevos conocimientos y a los recursos que favorecen el desarrollo de las capacidades de aprendizaje de las empresas. Asimismo, muestra que la centralidad en la red de colaboraciones, la experiencia en la gestión de estas relaciones y su diversidad tienen un efecto positivo sobre el crecimiento de las empresas (Powell y al., 1996, 1998). Powell y al. (1999) demuestran que la centralidad en la red de colaboraciones aumenta el desempeño económico de las empresas, medido a través de las patentes otorgadas y las ventas.

Estos resultados han sido corroborados por otros autores. Baum y al. (2000) demuestran, en su estudio de la industria biotecnológica en Canadá, que las empresas que establecen colaboraciones desde el momento de su creación tienen mejores retornos económicos. Salman y al. (2005) demuestran en su estudio sobre las empresas biotecnológicas en Quebec que la centralidad en la red indirecta de colaboraciones permite a las empresas acceder y aprovechar mejor los conocimientos de sus colaboradores directos. Owen-Smith y al. (2002) muestran por su parte las diferencias en la estructura de colaboraciones interorganizacionales ente los Estados Unidos y Europa. Según estos autores, los primeros beneficiarían de un sistema de relaciones heterogéneo, en donde la IA estaría más integrada a la investigación clínica que en Europa.

En el segundo grupo, encontramos los trabajos que se interesan a la relación entre los diferentes tipos de actividades (business model), y el "perfil colaborativo" o forma de organización elegido por la empresa (Pisano,1990, Oresingo, 2000, Niosi, 2003, Mangematin, 2003, Luukkonen, 2005, Saives y al., 2005). 
Pisano (1990) se interroga sobre los factores que entran en juego en la elección del modo de gestión de las empresas. Los costos de transacción asociados a la complejidad de los procesos de desarrollo, a la protección de la propiedad intelectual y a las exigencias reguladoras de los productos terapéuticos incitarían a las empresas a adoptar una forma integrada de gobernanza. Sin embargo, el autor señala que las formas colaborativas caracterizan este sector pues las necesidades financieras de las empresas limitan radicalmente su capacidad de adquirir las competencias necesarias para mantener su autonomía. Oresingo y al. (2000) estudian la relación que existe entre la naturaleza de las tecnologías desarrolladas por las empresas y las formas organizacionales de ID que implementan. En este trabajo distinguen dos lógicas de integración en la industria: una lógica de coespecialización, en la que las empresas más antiguas integran las más nuevas en una forma de división del trabajo; y una lógica transversal, en la que las empresas intentan modificar la división inter-generacional del trabajo.

Por su parte, Mangematin y al. (2003) estudian los business model de las empresas biotecnológicas en Francia. En su estudio diferencian dos tipos según la dimensión de los programas de investigación y el tipo de recursos y de interlocutores que las empresas necesitan. En el primero, las empresas se focalizan en mercados locales, venden servicios y encuentran el equilibrio económico rápidamente. En el segundo, las empresas apuntan a conquistar mercados mundiales, recaudan fondos de los CR y son deficitarias durante más tiempo. Para los autores, estos dos tipos de empresas no están en competencia directa: "Each type is a specific case, whit type A competing for markets and clients and type B for investors" (p. 635). Siguiendo esta tipología, Luukkonen (2005) muestra en su estudio sobre la industria biotecnológica en Finlandia, que las empresas de "drug discovery" tienden a establecer más colaboraciones inter-organizacionales que aquellas que tienen otros business model.

Por último, en el tercer grupo, encontramos los trabajos que estudian "el efecto de los clusters" o de la co-localización geográfica de las empresas biotecnológicas y sus interlocutores en la creación de empresas, la innovación, o el crecimiento económico (Aharonson y al., 2008, Audretsch y Stephan, 1996, Casper, 2007, Champenois, 2008, Chen, 2006, Coenen, 2004, Gertler, 2005, Gilding, 2008, Owen-Smith y al., 2004, Powell, 1996, Powell y al., 2002, Zuker y al., 1998). Ellos han establecido por ejemplo la importancia de las incubadoras (Lemarié y al., 2001) o de un elevado nivel de actividad científica local (Autant-Bernard y al., 2006, Zucker y al., 2001) en la creación de empresas. Otros estudios sugieren que 
la proximidad geográfica con empresas innovadores favorece las capacidades de innovación (Boufaden et al., 2005).

\subsection{Limitaciones del enfoque contractual}

Los trabajos que acabamos de evocar analizan exclusivamente las relaciones contractuales entre las empresas. Veremos en esta sección cómo la naturaleza de estas relaciones determina el tipo de análisis que puede realizarse. Mostraremos que las relaciones informales entre los empresarios son a menudo consideradas de manera implícita por estos estudios.

\subsubsection{El estudio de un medio social}

La mayoría de los trabajos sobre la industria biotecnológica adoptan un enfoque económico. La definición de su objeto de estudio permanece a menudo implícita, pero suele focalizarse en los intercambios comerciales. En este contexto, los trabajos de Powell parecen interesarse a las características sociales que estructuran esta industria. Nos apoyamos en estos trabajos, y en particular en las nociones de comunidad tecnológica y de reputación, para desarrollar nuestra crítica al enfoque contractual, pero nuestros argumentos son igualmente válidos para la inmensa mayoría de estudios sobre el sector (incluso si no todos reivindican una dimensión sociológica).

En uno de sus artículos fundadores (Powell, 1996), el autor anuncia la existencia de un mecanismo social de gobernanza que atañería los miembros de una misma comunidad tecnológica:

(...) membership in a common technological/intellectual community creates strong and visible mechanisms for peer based governance. As well as sharing the larger goal of advancing biomedical knowledge, and reaping the considerable rewards associated with such gains, participation in a research community affords the opportunity to monitor how participants behave in a wide range of settings, to discuss reputations with others, and to read their work in scientific journals. Pressures to publish, and thus reveal the latest advances are intense in this field. Thus discovery is open to all to evaluate. To reap the advantages of research, participants need to learn fast and collaborate effectively. Members of this community have ample opportunity to observe how individuals and organizations behave and learn about their reputations. The result of such sustained contact is that one's standing in the technological community shapes one's reputation for business practice (Powell, 1996, p. 211).

Los empresarios científicos compartirían ciertos objetivos e intereses al interior de una comunidad tecnológica. Se observarían mutuamente al tiempo que construyen y evalúan las reputaciones individuales y organizacionales. El autor afirma que las actividades científicas, en particular la publicación en revistas especializadas, determina la reputación vinculada a las prácticas económicas. Si bien es cierto que 
la mayoría de las empresas provienen del universo de la IA, a partir del momento en que comienza a gestionarse su creación, ésta entra en conflicto con otras lógicas de funcionamiento. Noisi, por ejemplo (2003, p. 743), destaca las dificultades encontradas por las empresas en sus relaciones con la IA: “( ...) university partners and gouvernement organisations had different timeframes and goals : researchers within the alliance wanted to publish, while companies preferred to keep information secret as long as possible". La publicación de artículos en revistas especializadas no es entonces necesariamente una norma para estos empresarios, aún cuando lo es para los científicos. Saives et al. (2005, p. 165-67) muestran igualmente en su investigación sobre las trayectorias de las empresas biotecnológicas, que el paso del sector académico al sector privado constituye una "ruptura teleológica" para los empresarios científicos. De una lógica de "reconocimiento intelectual" vinculada a la difusión de saberes a través de las publicaciones, estos deben adaptarse a una lógica de "reconocimiento económico" vinculada a la explotación de las patentes.

Estos trabajos muestran que las publicaciones son un indicador válido de las competencias en el sector de la IA. Éstas participan en la construcción de los status científicos en dicho sector, que es uno de los principales interlocutores de las empresas biotecnológicas. Estos dos sectores colaboran y entran en conflicto sistemáticamente. Es así que se han creado específicamente organizaciones en los centros de IA para manejar el paso de los proyectos de un universo al otro: se trata de las oficinas de transferencia tecnológica o de valorización. Estos organismos y las empresas biotecnológicas deben manejar en conjunto, además de las dificultades vinculadas al timing de las publicaciones, la negociación de los derechos de autor sobre las patentes. Sobre esta cuestión las "lógicas de acción" entre estos dos sectores pueden oponerse pues los riegos relativos al desarrollo de los descubrimientos científicos pueden no ser percibidos ni evaluados de la misma manera por las dos partes. De esta manera, incluso la "reputación económica" en la industria biotecnológica incluye la reputación científica de los actores. Estos ejemplos muestran que no es siempre posible transferir directamente el modo de funcionamiento, los valores o las normas del "mundo científico" al "mundo de la empresa".

Esto también es cierto para los otros principales interlocutores de las empresas. Las Big-Pharmas o les CR mantienen múltiples intercambios con las empresas biotecnológicas. Además, comparten ciertos intereses y valores, pero no se ven confrontados a las mismas dificultades, ni controlan las mismas incertidumbres. De hecho, las relaciones entre estos diferentes tipos de actores se ven marcadas por 
múltiples conflictos y negociaciones en los que el rol que juega cada actor lo sitúa en una identidad precisa, reconocida por sus pares e interlocutores. Desde una visión macroeconómica, la IA, las Big-Pharmas, los $C R$ y las empresas biotecnológicas forman parte de la misma filial industrial (la de los productos terapéuticos), pero de desde una perspectiva sociológica y organizacional, la noción de comunidad tecnológica definida por Powell (que corresponde de hecho a la definición económica utilizada por la mayoría de los estudios sobre el sector) subestima la complejidad y los conflictos socioeconómicos que caracterizan los intercambios entre estos diferentes tipos de actores.

\subsubsection{Naturaleza de las relaciones inter-organizacionales}

Como hemos mencionado, el autor analiza exclusivamente las relaciones contractuales (Powell, 1996). El cálculo de la centralidad y los años de experiencia en tanto que colaborador le permitieron construir una medida de reputación para las empresas ${ }^{5}$. Esta medida está correlacionada con el desempeño económico. Sin embargo, y pese a la importancia teórica y metodológica de estos resultados, estos no nos dicen mucho sobre los mecanismos sociales por los cuales los actores construyen y negocian sus reputaciones. Las nociones de reputación y de comunidad tecnológica tienen que ver exclusivamente con las actividades y resultados económicos puesto que éstas están descritas únicamente a través la observación de las relaciones contractuales fundadas sobre el intercambio de recursos comerciales. Esta mercantilización de los intercambios permite homogenizar los diferentes tipos de relaciones y analizar al mismo tiempo las empresas biotecnológicas, los centros IA, las Big-Pharmas, los CR los proveedores, etc. En consecuencia, la reputación, representada por el "perfil colaborativo" de las empresas, no es sino una característica económica, como la tasa de crecimiento, los efectivos o el tamaño del mercado objetivo. Ahora bien, en el análisis de los fenómenos inter-organizacionales, la simplificación analítica, que se refiere a la deducción sistemática de la reputación de los actores a partir de las relaciones contractuales, puede conllevar en ciertas condiciones a malos entendidos. Por un lado, porque los intercambios entre las personas no son económicamente evaluados ni encuadrados en su totalidad. Por otro lado, porque los actores perciben parcialmente no solamente la estructura social del medio en el que se mueven, sino también a menudo los resultados, los costos, la calidad y el sentido de las

\footnotetext{
5 “(...) number of years of experience with collaboration generates more external ties as firms develop a reputation for, and at, cooperation. The extensiveness of a firm's network is a strong predictor of survival."(Powell 1996, p. 208).
} 
relaciones que vinculan a los actores (ver la noción de endogénéisation en Lazega, 2008).

Un ejemplo sacado de nuestra investigación puede servirnos para ilustrar nuestra argumentación. Los ex-dirigentes de la empresa A benefician en sus actividades actuales de una reputación importante. Ésta está relacionada con el éxito financiero obtenido en la venta a una Big-Pharma de su empresa precedente. Sin embargo, simultáneamente, el medio de los empresarios en biotecnológica identifica y lamenta el fracaso industrial de los proyectos que fueron vendidos tan exitosamente. Este fracaso es percibido como una de las razones que han podido dañar la "credibilidad" de las empresas biotecnológicas francesas frente a sus principales clientes, las Big-Pharmas. En este ejemplo, la centralidad medida por el análisis de los contratos no tomaría en cuenta el resultado de la relación interorganizacional. Este resultado es sin embargo observado y evaluado por el conjunto de los actores. Considerar la complejidad de los juicios de los actores (juicios contradictorios, no fundados económicamente e incluso arbitrarios) permitiría, en nuestro ejemplo, explicar el desempeño actual de estos dirigentes quienes, pese a una buena reputación, no logran encontrar los socios que necesitan para desarrollar sus proyectos actuales.

La simplificación implementada en el enfoque contractual permite reconstituir la estructura económica de la industria y su evolución, pero no acceder a las múltiples formas de status que se derivan "de la existencia de varias concentraciones de recursos diferentes" (Lazega, 2003, p. 321), y a partir de las cuales el investigador puede intentar comprender come un colectivo resuelve los problemas (de regulación, de control, de aprendizaje) vinculados a la cooperación (Lazega, 2008).

Tomar en cuenta la posición social de los actores en una industria significa ir más allá de los intercambios comerciales. Nosotros defendemos la idea de que el estudio de su estructura social necesita la articulación de varios niveles de análisis y, en particular, del nivel inter-individual. La importancia de estas relaciones en las actividades de producción ha sido establecida en la industria textil (Uzzi, 1996), en la hotelería (Igram y Roberts, 2000) o en el medio de los cancerólogos (Lazega y al. 2006). Por el momento, ningún estudio ha examinado sistemáticamente las relaciones informales entre los empresarios científicos en el sector biotecnológico. Sin embargo, y como veremos a continuación, la necesidad de observar este tipo de lazos ha sido evidenciada por numerosos trabajos.

\subsubsection{Relaciones informales}


En su estudio sobre las formas organizacionales o de gestión implementadas en la industria biotecnológica, Pisano (1990) señala, entre los riesgos asociados a las estrategias de integración vertical, la partida de la empresa de los actores que fundaron los programas de investigación. La empresa que decide comprar otra con el objetivo de extender su portafolio de ID no cuenta necesariamente con las competencias necesarias para evaluar y dirigir los nuevos proyectos. En esto la empresa depende de los nuevos empleados, aunque no pueda ejercer un control realmente eficaz sobre ellos. El costo de transacción que se deseaba eliminar con la compra original se transforma así en una fuente de incertidumbre al interior de la empresa, debido al hecho de que son los investigadores los principales "activos" de la operación de adquisición. Casper (2007), en su estudio sobre las trayectorias profesionales, subraya las dificultades que deben enfrentar los dirigentes para integrarse en las empresas que han adquirido la suya. El autor muestra cómo los dirigentes de Hybritech, que rápidamente abandonaron la empresa que había comprado la suya, se llevaron consigo los recursos individuales que les permitirían crear otras empresas, al punto de ser considerados hoy como los fundadores del clúster de San Diego en los Estados Unidos. Sin hacer referencia explícita a las dificultades inter-individuales asociadas a los intercambios comerciales, estos autores hacen emerger el problema del control individual de ciertos recursos y su efecto en las relaciones inter-organizacionales.

Por su parte, en su estudio crítico sobre el peso de las alianzas organizacionales en la industria biotecnológica canadiense, Niosi (2003) constata la importancia de las relaciones con los $\mathrm{CR}$ en la "credibilidad de las empresas al interior de la comunidad" ${ }^{\prime}$. Mangematin y al. (2003), en su estudio sobre las trayectorias de las empresas biotecnológicas francesas, observa el mismo fenómeno, pero esta vez es la reputación en el mundo científico la que haría creíble un proyecto evaluado por los inversionistas en $\mathrm{CR}^{7}$. Esta cuestión de la credibilidad de los empresarios es recurrente en los estudios sobre esta industria, ya que por definición no se cuenta con la fiabilidad de las tecnologías que se pretende desarrollar. El equipo dirigente es a menudos el único "activo" que los potenciales socios de la empresa pueden evaluar. Los trabajos evocados sugieren que la credibilidad de un proyecto está relacionada con la reputación del empresario, pero no dicen nada sobre la manera en la que los actores la evalúan. De manera explícita, los trabajos de Powell y al.

\footnotetext{
6 “(...) one-third of the companies declared having acquired credibility vis-a-vis the biotechnology community - as an intangible externality- from their venture capitalists." (Niosi, 2003, p. 743).

7 "(...) The visibility of the scientist in the academia community plays a key part as regards venture capital. It gives credibility to the scientific project of the firm." (Mangematin et al., 2003, p. 634).
} 
(1996) señalan cuán importante es tomar en consideración las relaciones interindividuales:

In our view, collaborations in high-tech industries typically reflect more than just a formal contractual exchange. When the first author presented the chief executive officer of Centocor whit a list of his firm's formal agreements, he observed that it was "the tip of the iceberg [...]". Beneath most formal ties, then, lies a sea of informal relations. Many alliances - no matter what their ostensible function- reflect a relationship that carries benefits beyond the particular exchange designated in a formal agreement. (Powell et al. 1996, p. 118)

Para estos autores, las relaciones contractuales son en parte el indicador de las relaciones informales, pero en sus trabajos éstas últimas son siempre supuestas. Por lo tanto, el investigador no puede formular hipótesis en este nivel de análisis y debe contentarse con cartografiar las relaciones económicas entre los actores. Así, por construcción, el enfoque contractual no toma en consideración los juicios de los actores sobre las relaciones. Los resultados de las relaciones, los compromisos personales o las relaciones con terceros son elementos sistemáticamente excluidos.

Por último, los trabajos sobre la co-locación industrial estudian igualmente de manera implícita el rol de las relaciones inter-individuales. La transmisión de conocimientos tácitos (Jaffe y al., 1993) es posible gracias a las relaciones personales. De este modo, la problemática de los clusters reposa sobre la idea de que, compartiendo un entorno institucional y físico, los actores tienen más posibilidades de entrar en relación. Medir la proximidad geográfica como un factor susceptible de aumentar las relaciones inter-organizacionales, el desempeño científico o económico, constituye un intento que busca tomar en cuenta el peso de las relaciones inter-individuales en el acceso a las oportunidades que se presentan y que pueden capturar los actores.

\subsection{Relaciones inter-individuales y cooperación entre competidores}

En este artículo sugerimos que la dificultades que atañen el enfoque contractual pueden ser superadas a través de la observación de las relaciones sociales a nivel inter-individual (ver por ejemplo Pina-Stranger, 2009, Pina-Stranger y Lazega, 2010). El análisis de este tipo de relaciones permite en particular estudiar cómo los miembros de un sector de producción cooperan aún cuando son competidores (White, 1981, Lazega y Mounier, 2002). A este nivel de análisis, no se toma solamente en cuenta los intercambios económicos puesto que se considera que los intercambios sociales participan igualmente en la estructuración de las actividades de producción. Las experiencias, las identidades, los valores o el status social constituyen también recursos que se crean y circulan entre los actores, y que se 
articulan a los recursos económicos. La racionalidad de los actores incluye esta dimensión simbólica, que les permite contextualizar y politizar sus relaciones (Lazega, 2003) para, por ejemplo, elegir su grupo de referencia o jerarquizar las autoridades que “luchan” por legitimar su dominación (Lazega, 1992).

Considerar las dimensiones simbólica y estratégica de los intercambios permite examinar cómo los actores manejan sus relaciones para acceder a los recursos, pero también la manera en la que se negocian las condiciones de intercambio, se reivindican ciertos valores o se delimitan las fronteras sociales entre diferentes grupos (Lazega, 2003). La politización de los intercambios es la base sobre la que se desarrollan los mecanismos de asignación de recursos (económicos, simbólicos, políticos, sociales, etc. ).

La politización de los intercambios es la base de los mecanismos de asignación de los recursos en la medida en que, para participar en el sistema de intercambio, los actores deben respetar una serie de compromisos frente al colectivo que integran (Lazega, 2003). El estudio de las interdependencias creadas por este juego micropolítico (Reynaud, 1989, Lazega, 1992) permite describir y analizar con todo detalle los mecanismos de la acción colectiva, como los procesos de regulación, de control social, de integración y exclusión, de aprendizaje, de innovación o de institucionalización (Lazega, 2008).

Ahora bien, para poner al día esta dimensión política de los intercambios, es necesario observar las relaciones personales que mantienen los actores. Esto a fin por ejemplo de disociar la reputación económica, medida a través de las relaciones contractuales, de los status sociales, medidos a través del intercambio de recursos a nivel inter-individual.

Por ello, contrariamente al enfoque contractual donde los distintos tipos de interlocutores participan en la red como si fuera un medio homogéneo, la definición de los recursos intercambiados entre los actores individuales permite analizar de manera detallada la estructura y las dinámicas sociales con el fin de, por ejemplo, disociar los modos de valorización de la IA (publicaciones) de las los de la industria biotecnológica (patentes).

Por último, observar las elecciones relacionales de los actores a nivel interindividual permite no sólo acceder a los intercambios informales supuestos en el enfoque contractual, sino también considerar la evaluación que los actores hacen de las posiciones, resultados y relaciones de los otros actores.

\subsubsection{Pregunta de investigación}


En un contexto de producción inter-organizacional como la industria biotecnológica, el análisis de los intercambios comerciales ha permitido trazar sus fronteras y su modo de funcionamiento macroeconómico. Pero la naturaleza de dichas relaciones no ha permitido analizar los procesos sociales que favorecen la cooperación en este medio (Lazega, 2001). Los recursos intercambiados a través de las relaciones sociales son costosos y difíciles a encontrar ya que se refieren a conocimientos tácitos, experiencias recientes, que aún no se estabilizan (Jaffe y al., 1993). En la industria del conocimiento, la pregunta por la pertinencia de esta información es decisiva y problemática. La politización de los intercambios permite al colectivo atribuir a los recursos intercambiados una "firma", una autoridad a partir de la cual cada actor puede juzgar su pertinencia (Lazega, 1992).

El alto nivel de exigencias científica, financiera y de regulación que caracterizan la industria biotecnológica impide la rutinización de las tareas. En consecuencia, son las relaciones a menudo entre competidores que permiten acceder a los "recursos adecuados".

La investigación que hemos diseñado tiene por objetivo estudiar la manera en que los dirigentes de biotecnología Biotech en Francia se asocian para hacer más accesibles estos recursos. ¿Cómo estos empresarios científicos, competidores en el acceso a los recursos más escasos, cooperan y se coordinan con el fin de incrementar colectivamente sus posibilidades de éxito?

\subsubsection{Hipótesis}

Para responder a estas cuestiones, nos hemos interesado en las relaciones personales que mantienen los dirigentes de empresas de biotecnología en Francia. El estudio de estas relaciones constituye a la vez un complemento necesario al enfoque contractual y un nuevo objeto de estudio para este sector de actividad. Si el análisis de las relaciones contractuales estableció las formas y la evolución de la industria biotecnológica a distintos niveles (clúster industrial, regional, por país y continentes), la existencia de un sistema de intercambio informal entre los empresarios científicos en esta industria no se ha estudiado directamente ${ }^{8}$. En consecuencia, la primera y más general hipótesis de nuestro estudio se refiere a la existencia de este sistema de intercambio:

\footnotetext{
${ }^{8}$ Los trabajos de Casper (2007) muestran la existencia de un medio social conformado por los dirigentes de Biotecnología en San Diego, pero sólo observa las relaciones de afiliación entre estos actores y sus empresas. Los datos de los que dispone no le permiten examinar de manera directa las redes de intercambio de recursos.
} 
H1: La industria biotecnológica en Francia es un sector de actividad que reposa sobre un sistema de intercambio informal cohesivo, en el seno del cual los actores mantienen distintos tipos de relaciones.

Las relaciones observadas conforman una estructura social en la cual podemos observar la posición que ocupan los actores en el sistema de intercambio. El número de elecciones relacionales recibidas por los actores (indegree) permite calcular distintas medidas de centralidad a partir de las cuales es posible identificar varios subgrupos de actores. En función de la posición que estos ocupan en la estructura, se ven beneficiados por distintas formas de intercambio. En este sentido, la centralidad determina en parte el acceso a los recursos (así como también el costo vinculado al mantenimiento de esta posición, Uzzi, 1997).

Según la famosa intuición de Blau (1964), para quien los actores comparten sus conocimientos en la medida en que obtienen en contraparte el reconocimiento de su status y su autoridad epistémica, nuestra segunda serie de hipótesis explora la morfología global del sistema de intercambio en términos de estratificación social jerárquica:

H2: La estructura social de este sistema de intercambio exhibe una división jerárquica donde el centro se diferencia de la periferia por:

H2a) una mayor densidad en las relaciones;

H2b) una mayor multiplicidad en las relaciones;

H2c) y una mayor reciprocidad en las relaciones.

Otros factores determinan también la manera en que los actores intercambian recursos. Nuestras últimas hipótesis exploran, en función de los atributos de los empresarios, la existencia de normas que orientan los intercambios relacionales en este medio. El primer atributo se refiere a la condición de fundador o de no fundador del dirigente:

H3a: Los dirigentes que han sido fundadores de una empresa en el sector de la Salud humana son más activos en el sistema de intercambio.

H3b Los dirigentes que han sido fundadores de una empresa en el sector de la Salud humana benefician de un status social más importante en el sistema de intercambio.

El trabajo de valorización de los descubrimientos científicos que realizan las empresas biotecnológicas implica la "transferencia de tecnologías" del sector público, cuyo trabajo se focaliza en la investigación científica, hacia el sector 
privado, cuyo trabajo se focaliza en el desarrollo industrial. El paso de una "lógica de investigación" hacia una "lógica de mercado" (Saives y al. 2005) presenta una serie de obstáculos que los empresarios deben superar. Estos deben tener en cuenta, además de las dificultades científicas, las dificultades clínicas, de regulación y económicas. En este contexto, nuestra última hipótesis explora cómo estas dificultades, personificadas por el cargo de los actores en las empresas, determinan el intercambio de recursos entre los dirigentes.

H3C: Existe un déficit en el sistema de intercambio de recursos entre los dirigentes que pertenecen al grupo de los Directores científicos y el grupo de los Directores financieros.

La sección siguiente presenta el terreno y la investigación que realizamos para probar estas hipótesis.

\section{Un estudio de caso: los empresarios científicos en la industria biotecnológica en Francia}

Las hipótesis que acabamos de formular tienen por objetivo general establecer la existencia de un sistema de intercambio informal cohesivo en la industria biotecnológica francesa, así como algunas de las características de su modo de funcionamiento. Con el fin de probar nuestras hipótesis, y de justificar la necesidad de un enfoque inter-individual en el análisis de las relaciones interorganizacionales, diseñamos y realizamos una investigación sobre una subpoblación de empresarios científicos en biotecnología.

\subsection{Estudio de campo y subpoblación}

El diseño de la investigación contempla dos etapas. La primera consistió en una investigación cualitativa destinada a identificar y delimitar a la población objeto de nuestro estudio. Se realizaron más de 30 entrevistas no estructuradas y se asistió a varias jornadas de observación no participativa.

Este estudio de campo permitió definir un conjunto de 96 PYME innovadoras en biotecnología, dedicadas a la ID en el sector de la salud humana en Francia. Excluimos de nuestra población a más de a 150 empresas según los siguientes criterios: a) no trabajar en el sector de la salud humana; b) realizar actividades de servicio sin una tecnología propietaria; c) realizar actividades de consejo; d) no recaudar fondos; e) pertenecer a una Big-Pharma. Las empresas que dejamos en nuestra población comparten al menos tres características: a) trabajan en el ámbito de las Ciencias de la vida, es decir, en la comprensión y/o la modificación de los 
organismos vivos; b) fueron objeto de inversiones privadas y/o públicas de un importe superior a $500 \mathrm{~K} €$; c) y gastan en ID al menos $15 \%$ de sus costos totales.

En representación de estas empresas, incluimos en nuestra población a los miembros de la dirección ejecutiva según los siguientes criterios: a) fundadores; b) Presidente $\mathrm{y} / \mathrm{o}$ Director General; c) director científico, financiero, médico y/o farmacéutico. Según el nivel de desarrollo de la empresa, incluimos entre 1 y 4 dirigentes. La población original contaba con 229 individuos pertenecientes a 96 empresas.

A continuación, concebimos y probamos un cuestionario destinado a colectar información sobre las características de los actores y sus relaciones con los otros empresarios.

En la segunda etapa de nuestra investigación, contactamos y entrevistamos a los empresarios. A medida que obteníamos la información, los integrábamos en nuestra base de datos (matriz relacional). Esto nos permitió ir definiendo, en función de las respuestas ya obtenidas, cada vez más pertinentemente a los empresarios que debíamos entrevistar en cada empresa. Excluimos a 8 empresas de nuestra población pues no cumplían con los criterios de inclusión. Se excluyeron también a 65 actores que no eran representativos de su empresa en este medio.

Condujimos 125 entrevistas cara a cara y 13 cuestionarios fueron recibidos por correo electrónico. Dejamos en nuestra población final 164 actores y 88 empresas, 79 de las cuales están representadas por el cuestionario individual. La información relativa a los 26 actores que no respondieron el cuestionario se recolectó por otros medios, a excepción de los datos relaciones (elecciones emitidas o outdegree). El Cuadro I recapitula el número de actores que integramos en las distintas etapas de nuestra investigación a nivel individual y organizacional.

\begin{tabular}{l|c|c|c|c|c}
\multicolumn{1}{c|}{ Nivel } & $\begin{array}{c}\text { Población } \\
\text { original }\end{array}$ & $\begin{array}{c}\text { Población } \\
\text { entrevistada }\end{array}$ & $\begin{array}{c}\text { Población } \\
\text { final }\end{array}$ & $\begin{array}{c}\text { Datos } \\
\text { faltantes }\end{array}$ & $\begin{array}{c}\% \text { de datos } \\
\text { faltantes }\end{array}$ \\
\hline Individual & 229 & 138 & 164 & 26 & $15,85 \%$ \\
\hline Organizacional & 96 & 78 & 88 & 10 & $\mathbf{1 1 , 3 6 \%}$
\end{tabular}

Cuadro I. Población a nivel individual (empresarios) y organizacional (empresas) por etapa de investigación

Nota: El porcentaje más bajo de datos que faltan a nivel organizacional se explica por el hecho de que dejamos en nuestra población final a algunos actores que trabajaban en una empresa ya representada en nuestra población por otro dirigente entrevistado.

\subsection{Datos y recursos intercambiados}

En el Cuadro II presentamos las características de los empresarios de nuestra población final, en función de los tres tipos de actividades (business model) 
representados: desarrollo de productos terapéuticos; desarrollo de productos de diagnóstico; y actividades de servicio a la investigación.

\begin{tabular}{r|c|c|c|c} 
& \multicolumn{2}{c}{ Tipo de actividad } \\
Atributos & Productos & Diagnóstico & Servicio & Total \\
\hline $\mathrm{N}^{\circ}$ de empresas/ $\mathrm{N}^{\circ}$ no representadas por cuestionario & $58 / 6$ & $8 / 0$ & $22 / 4$ & $88 / 10$ \\
$\mathrm{~N}^{\circ}$ de actores/no entrevistados & $107 / 15$ & $19 / 2$ & $38 / 9$ & $164 / 26$ \\
\hline$\%$ de No fundadores & $\mathbf{4 2 \%}$ & $32 \%$ & $34 \%$ & $39 \%$ \\
\hline$\%$ Origen Investigación académica & $22 \%$ & $\mathbf{4 7 \%}$ & $\mathbf{4 5 \%}$ & $30 \%$ \\
$\%$ Origen Industria Salud & $\mathbf{5 7 \%}$ & $32 \%$ & $34 \%$ & $49 \%$ \\
$\%$ Otro origen & $21 \%$ & $21 \%$ & $21 \%$ & $21 \%$ \\
\hline$\%$ formación inicial económica & $22 \%$ & $11 \%$ & $11 \%$ & $18 \%$ \\
$\%$ formación económica & $\mathbf{4 9 \%}$ & $\mathbf{4 2 \%}$ & $\mathbf{3 4 \%}$ & $45 \%$ \\
\hline Edad promedio & 49,48 & 46,00 & 44,86 & 46,78 \\
\% Mujeres & $10 \%$ & $11 \%$ & $11 \%$ & $10 \%$
\end{tabular}

Cuadro II. Principales características de los empresarios por tipo de actividad.

Más del $65 \%$ de las empresas representadas en nuestra población (58 de 88) desarrollan productos terapéuticos. Esto se debe a los criterios de selección presentados anteriormente, en particular, el acceso a los recursos financieros. Las empresas de servicio y diagnóstico recurren menos a grandes recaudaciones de fondos (Mangematin y al., 2003). El número de empresarios no fundadores es más elevado en la categoría "Producto" (42\%) que en los otros tipos de actividad. El origen profesional de los dirigentes corresponde al sector de actividad donde el actor ha pasado la mayor parte de su carrera.

Los empresarios de las categorías "Diagnóstico" y "Servicio" vienen principalmente del mundo de la IA ( $47 \%$ y $45 \%$ respectivamente), mientras que los de la categoría "Producto" vienen más bien de la industria de la Salud humana ( $57 \%$ vienen de las Big-Pharmas y de las empresas biotecnológicas). Más de tres cuartos de los empresarios de la categoría "Producto" (83\%) realizaron estudios iniciales científicos, pero casi la mitad $(49 \%)$ ya siguió una formación en economía (formación inicial o continua). Este porcentaje es menos importante para los otros empresarios, en particular, los de la categoría "Servicio" (34\%). Estos últimos son los más jóvenes (44 años en promedio). Por último, las mujeres están infrarrepresentadas en todas la categorías (10\%).

En lo que se refiere a las relaciones que mantienen los empresarios entre ellos, hemos reconstituido tres tipos de redes de intercambio: de Discusión sobre la dirección (management) de la empresa, de Amistad y de Consejo. El primero se refiere al intercambio general de información sobre la situación industrial del sector y sobre temas corrientes como el Crédit d'Impôt Recherche, el statut de Jeune Entreprise Innovante, los prestadores de servicios, etc. 
La red de Amistad se reconstituyó a partir de las respuestas otorgadas a la siguiente pregunta: ¿“En esta lista de empresarios, podrían decirnos cuáles son las personas que son sus amigos? Piense en un amigo como alguien con quien permanecería en contacto aunque cambiase radicalmente de oficio". Así pues, el "recurso amistad" del que esta red da cuenta, se refiere a las relaciones que los actores esperan poder mantener a largo plazo.

La tercera red se refiere a las relaciones de Consejo. Definimos tres tipos principales de actividades realizadas por los empresarios. Estas actividades corresponden a la gestión del trabajo de colaboración que mantienen con sus principales socios: Ios Institutos IA, las Big-Pharmas, y los inversionistas en CR. Los empresarios que estudiamos deben gestionar las relaciones con estos tres socios durante toda la vida de la empresa. Los institutos de IA son a menudo el lugar de origen de las empresas, ya que de allí provienen los científicos fundadores. Son el socio clave en el periodo de fundación de las empresas.

Por su parte, los inversionistas en CR proporcionan el dinero que permite a los empresarios sacar el proyecto científico del laboratorio académico y desarrollarlo en una estructura privada. Los CR se convierten en copropietarios de la empresa y ocupan a menudo un puesto en su consejo de administración. Este interlocutor es clave para el crecimiento rápido que deben implementar las empresas. Por último, las Big-Pharmas constituyen uno de los principales clientes del Biotech. Ellas compran los productos en desarrollo o firman contratos de colaboración con las empresas biotecnológicas. Son el socio clave en las fases avanzadas de desarrollo de las empresas.

\section{Resultados y discusión}

Presentamos los resultados de nuestra investigación en tres secciones. La primera se refiere a la existencia de un sistema de intercambio general entre los dirigentes en biotecnología en el que circulan distintos recursos. El segundo da cuenta de la estructura este sistema de intercambio. Por último, la tercera sección examina el comportamiento relacional de los empresarios en un contexto inter-organizacional.

\subsection{Un sistema de intercambio en el que circulan diversos recursos}

Hemos observado el conjunto de las relaciones que mantienen estos empresarios.

El Cuadro III presenta las principales características de las tres redes de intercambio reconstituidas a nivel inter-individual e inter-organizacional. Construimos el nivel organizacional haciendo la síntesis de las elecciones relacionales emitidas por los miembros de una misma empresa. A continuación, 
borramos las relaciones intra-organizacionales con el fin de analizar exclusivamente las relaciones entre las empresas. La red de consejo corresponde a la síntesis de las respuestas otorgadas en los 3 tipos de recursos definidos (IA, Big-Pharmas y CR). Hicimos esta síntesis "por persona", es decir consideramos las elecciones relacionales sin tener en cuenta el número de recursos para los cuales un actor solicitó a otro.

\begin{tabular}{r|cc|cc|cc} 
Redes a nivel & \multicolumn{2}{|c|}{ Todas las relaciones } & \multicolumn{2}{c|}{ Relaciones recíprocas } & \multicolumn{2}{c}{ Indegree } \\
\cline { 2 - 7 } Inter-individual (164) & \multicolumn{2}{|c|}{ arcs + edges Densidad } & $\mathrm{N}^{\circ}$ edges & $\%$ & Promedio Mediana \\
\hline Discusión sobre management & 1947 & $\mathbf{0 , 1 0}$ & 671 & $\mathbf{3 4 \%}$ & $\mathbf{1 6 , 0}$ & 12,0 \\
Consejos IA-PH-CR & 1039 & $\mathbf{0 , 0 5}$ & 192 & $\mathbf{1 8 \%}$ & 7,5 & 4,5 \\
Amistad & 521 & 0,03 & 149 & $\mathbf{2 9 \%}$ & 4,1 & 3,0 \\
Redes a nivel Inter-organizacional (88) & & & & & & \\
\hline Iiscusión sobre management & 1160 & $\mathbf{0 , 2 1}$ & 426 & $\mathbf{3 7 \%}$ & $\mathbf{1 8 , 0}$ & 16,0 \\
Consejos IA-PH-CR & 688 & $\mathbf{0 , 1 1}$ & 133 & $\mathbf{1 9 \%}$ & 9,3 & 7,0 \\
Amistad & 232 & 0,05 & 90 & $\mathbf{3 9 \%}$ & 6,1 & 5,5
\end{tabular}

Cuadro III. Principales características de las redes de intercambio a nivel inter-individual e interorganizacional.

Nota: Las redes de Discusión sobre el management, de Consejo y Amistad son examinadas a nivel interindividual, que cuenta con 164 empresarios, e inter-organizacional, que cuenta con 88 empresas. La densidad y el promedio de las elecciones recibidas disminuyen, mientras que el porcentaje de relaciones recíprocas es más importante en la red de Amistad que en el de Consejo.

Las 88 empresas incluidas en nuestro estudio fueron seleccionadas principalmente en función del tipo de actividad que realizan. El único criterio "relacional" utilizado fue la afiliación a un mismo sector de actividad y a un mismo territorio (Francia).

Sin embargo, el análisis de las características generales de las redes reconstituidas nos permite constatar la existencia de un sistema de relaciones extraordinariamente activo y extendido. La densidad de la primera red indica que un $10 \%$ de las relaciones posibles a nivel inter-individual, y un $21 \%$ de las relaciones posibles a nivel inter-organizacional se efectuaron. Más de un tercio de las relaciones en esta red son recíprocas (34\% y 37\% respectivamente en los dos niveles de análisis). El nivel de actividad puede ser apreciado por el número de elecciones recibidas (indegree): en esta primera red, los actores han sido elegidos en promedio 16 veces. El sistema de intercambio es general en la medida en que ningún actor está completamente aislado y, en promedio, cada empresa está en relación con 18 otras empresas.

Todos los valores son más bajos en las redes de Consejo y Amistad, ya que implican el intercambio de recursos más escasos, y se solicita más el compromiso y 
la inversión relacional de los actores. No obstante, nuestros resultados ponen de manifiesto que los empresarios intercambian distintos tipos de recursos, comparten información sobre las estrategias de gestión, intercambian consejos sobre sus principales interlocutores y tejen lazos de amistad.

La red de Consejo es menos densa que la primera ( $5 \%$ y $11 \%$ en los dos niveles de análisis). Esto indica una mayor especialización de los recursos intercambiados: es más fácil encontrar un interlocutor para intercambiar información genérica sobre la gestión de una empresa biotecnológica que encontrarlo para solicitarle información relativa a una de las problemáticas específicas representadas por la red de Consejo. Ésta presenta también el porcentaje de reciprocidad más bajo de las 3 redes (19\%), lo que, como veremos a continuación, puede indicar la existencia de una distribución jerárquica de los recursos intercambiados. Por su parte, la red de Amistad se caracteriza por la densidad más baja de las 3 redes $(0,03)$ y por un elevado porcentaje de reciprocidad (29\% y 39\% en los dos niveles de análisis). La distribución de este recurso es en este sentido menos jerárquica que la de la red de Consejo, aunque la baja densidad de la red sugiere que se trata del recurso más escaso de los tres observados.

Las dos proposiciones de nuestra primera hipótesis se ven confirmadas por estos resultados: los dirigentes de las empresas biotecnológicas mantienen a nivel interinter-individual e inter-organizacional numerosas relaciones y múltiples.

\subsection{Una estructura social jerárquica}

Para comprobar nuestra segunda hipótesis, exploramos la estructura general de la red de Consejo y de la red de Amistad. En primer lugar fusionamos estas dos redes con el fin de llevar a cabo un análisis de input k-cores en el que observamos exclusivamente las elecciones recibidas por los actores (ver Nooy y al., 2005, p. 70; o Wasserman, 1994, p. 266). Observar exclusivamente las elecciones recibidas (indegree) es una manera de manejar los datos faltantes en este tipo de análisis. El análisis de input k-cores permitió definir a 5 grupos de actores. Enseguida, volvimos a tomar separadamente las dos redes y estudiamos las relaciones al interior y entre los grupos. Los resultados se presentan en el Cuadro IV. 


\begin{tabular}{|c|c|c|c|c|c|c|}
\hline Red de Consejo & Grupo - 1 (24) & Grupo -2 (29) & Grupo - 3 (34) & Grupo - 4 (37) & Grupo - 5 (40) & Total outdegree \\
\hline Grupo-1 & $57 \%$ & $25 \%$ & $10 \%$ & $7 \%$ & $2 \%$ & 366 \\
\hline Grupo - 2 & $47 \%$ & $28 \%$ & $16 \%$ & $7 \%$ & $3 \%$ & 279 \\
\hline Grupo - 3 & $42 \%$ & $21 \%$ & $22 \%$ & $11 \%$ & $4 \%$ & 235 \\
\hline Grupo -4 & $39 \%$ & $22 \%$ & $16 \%$ & $18 \%$ & $4 \%$ & 178 \\
\hline Grupo - 5 & $41 \%$ & $17 \%$ & $16 \%$ & $9 \%$ & $16 \%$ & 170 \\
\hline Total choix reçus & $47 \%$ & $23 \%$ & $15 \%$ & $10 \%$ & $5 \%$ & 1228 \\
\hline \multicolumn{7}{|l|}{ Red de Amistad } \\
\hline Grupo - 1 & $54 \%$ & $26 \%$ & $12 \%$ & $5 \%$ & $3 \%$ & 215 \\
\hline Grupo - 2 & $34 \%$ & $34 \%$ & $17 \%$ & $10 \%$ & $5 \%$ & 175 \\
\hline Grupo-3 & $26 \%$ & $19 \%$ & $31 \%$ & $18 \%$ & $6 \%$ & 125 \\
\hline Grupo - 4 & $24 \%$ & $15 \%$ & $28 \%$ & $26 \%$ & $7 \%$ & 85 \\
\hline Grupo - 5 & $23 \%$ & $14 \%$ & $11 \%$ & $7 \%$ & $44 \%$ & 70 \\
\hline Total indegree & $36 \%$ & $24 \%$ & $19 \%$ & $12 \%$ & $9 \%$ & 670 \\
\hline
\end{tabular}

Cuadro I V. Porcentajes de las elecciones emitidas al interior y entre los grupos en las redes de Consejo y de Amistad a nivel inter-individual.

Nota: Los porcentajes indican la proporción de las relaciones emitidas por el conjunto de los actores de los 5 grupos (eje vertical) en dirección de cada grupo (eje horizontal), en función del número total de elecciones emitidas por sus miembros (Total outdegree). Los valores disminuyen del grupo-1 al grupo-5, pero esta tendencia es menos fuerte en la red de Amistad.

Como vimos en la sección anterior, en las redes de Consejo y Amistad se observan menos relaciones que en la red de Discusión. Los actores eligen más cuidadosamente a sus interlocutores para estos tipos de intercambios ya que deben invertir y comprometerse más para mantener estas relaciones. El costo vinculado al acceso, al mantenimiento y a la pertinencia de estas relaciones se refleja en el comportamiento de los actores a través de una mayor selectividad a la hora de elegir a un interlocutor. La distribución de las elecciones emitidas en los distintos grupos refleja en parte este proceso de selección. Los actores más elegidos ocupan una posición más central con relación a aquellos que han lo han sido menos. Esto les permite beneficiarse más de los recursos y oportunidades que circulan en el sistema de intercambio.

En la red de Consejo, los miembros del grupo- 1 son los principales destinatarios de las elecciones relacionales: reciben casi la mitad (47\%) de las elecciones emitidas por el total de actores (Cuadro IV). El grupo-2 es el segundo más elegido por los otros grupos (23\%) a excepción del grupo-3. El número de elecciones recibidas por el grupo-3 al -5 disminuye sistemáticamente en los valores totales, pero los grupos - 4 y -5 tienen más relaciones al interior de su grupo que entre ellos ( $18 \%$ y $16 \%$ respectivamente).

En la red de Amistad, los miembros del grupo- 1 siguen siendo los más elegidos (36\%). Esto se debe principalmente a las relaciones al interior del grupo-1 (54\%), y a la mayor actividad de sus miembros que concentran casi a un tercio de las elecciones emitidas ( 215 sobre 670 ). Los miembros de los grupos-2, - 3 y -5 eligen en primer lugar a los miembros de su mismo grupo $(34 \%, 31 \%$ y $44 \%$ de las 
elecciones emitidas respectivamente), y en segundo lugar a los miembros del grupo- 1.

Representamos estos resultados en la Figura I. El tamaño de los nodos es proporcional al número de elecciones recibidas por los actores en las redes de Consejo y Amistad fusionadas.

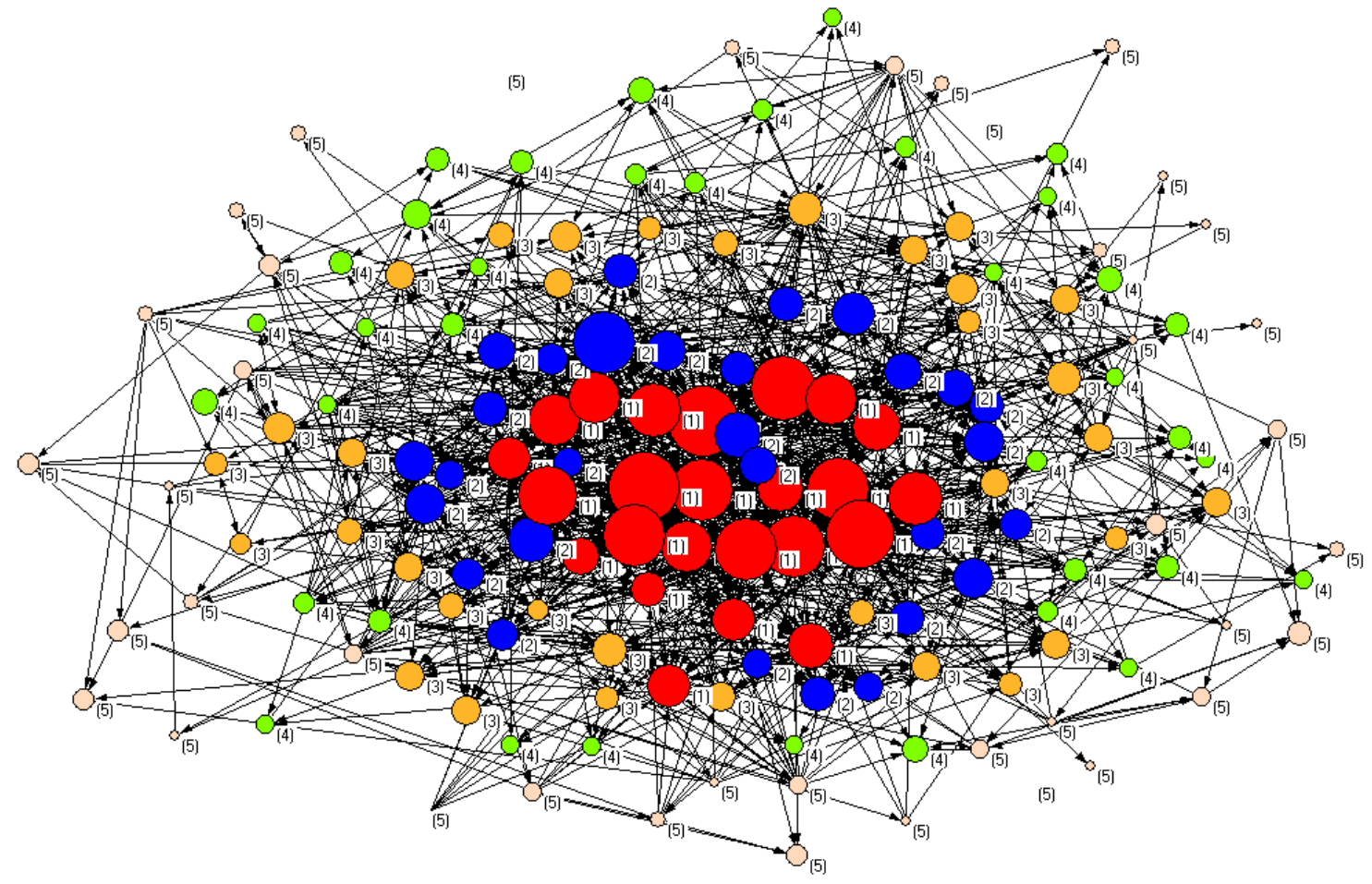

Figura I. Grafo de las relaciones entre los empresarios en las redes de Consejo y Amistad por grupo según el análisis Input k-cores et por número de elecciones recibidas.

Nota: El color y la cifra entre paréntesis indican al grupo al cual el actor pertenece (de 1 a 5). El tamaño de los nodos es proporcional al número de elecciones recibidas por los actores en las redes de Consejo y Amistad.

La estructura general que emerge del análisis de estas dos redes confirma la principal proposición de nuestra segunda hipótesis: la estructura social del sistema de intercambio sigue una distribución jerárquica en la cual es posible distinguir un centro (grupo-1) y varios grupos concéntricos (del grupo-2 al -5). No obstante, como veremos a continuación, las relaciones de amistad parecen escapar en parte a esta lógica.

Estos análisis puedes profundizarse ya que, una vez encontrada la estructura jerárquica, es posible explorar las formas relacionales que caracterizan los distintos grupos. Para esto, hemos calculado para cada uno de ellos la densidad y la proporción de relaciones recíprocas y múltiples. La densidad es la proporción de relaciones observadas con respecto al número total de relaciones posibles. El porcentaje de relaciones recíprocas se calculó en función del total de vínculos 
únicos entre los actores, es decir, sin contabilizar las relaciones recíprocas dos veces. Por último, consideramos una relación como múltiple cuando un actor ha elegido a un mismo interlocutor para el intercambio de los dos recursos analizados: el Consejo y la Amistad. El Cuadro $V$ recapitula los resultados obtenidos en estos análisis.

\begin{tabular}{l|c|c|c|c|c} 
Red de Conejo y Amistad & Grupo-1 (24) & Grupo -2 (29) & Grupo - 3 (34) & Grupo -4 (37) & Grupo -5 (40) \\
\hline Densidad & $\mathbf{4 1 \%}$ & $13 \%$ & $6 \%$ & $3 \%$ & $\mathbf{3 \%}$ \\
$\%$ relaciones múltiples & $\mathbf{4 3 \%}$ & $27 \%$ & $34 \%$ & $20 \%$ & $\mathbf{4 4 \%}$ \\
$\%$ relaciones recíprocas & $45 \%$ & $28 \%$ & $24 \%$ & $18 \%$ & $21 \%$ \\
Red de Consejo & & & & & \\
\hline Densidad & $38 \%$ & $10 \%$ & $5 \%$ & $2 \%$ & $2 \%$ \\
$\%$ relaciones recíprocas & $\mathbf{4 3 \%}$ & $13 \%$ & $21 \%$ & $10 \%$ & $12 \%$ \\
Red de Amistad & & & & & \\
\hline Densidad & $21 \%$ & $7 \%$ & $3 \%$ & $2 \%$ & $2 \%$ \\
\% relaciones recíprocas & $\mathbf{3 1 \%}$ & $36 \%$ & $22 \%$ & $16 \%$ & $29 \%$
\end{tabular}

Cuadro V. Densidad, reciprocidad y multiplicidad de las relaciones intra-grupo en las redes de Consejo y Amistad a nivel inter-individual.

Nota: La "Red de Consejo y Amistad" es una fusión destinada a calcular la proporción de relaciones múltiples que existe dentro de los grupos. Los valores de las densidades disminuyen del grupo- 1 al - 5 en las 3 redes. Los valores de las relaciones recíprocas no siguen la misma lógica en el análisis de las redes tomadas independientemente.

El análisis de las relaciones de Consejo y Amistad fusionadas nos permite calcular la proporción de relaciones múltiples en cada grupo. Si bien el grupo- 1 y el grupo- 5 tienen un porcentaje similar de relaciones múltiples ( $43 \%$ y $44 \%$ respectivamente), la densidad de las relaciones del primero (41\%), comparada a la del grupo-5 (3\%), indica la existencia de un fenómeno de multiplicidad más significativo. Por otra parte, la distribución de las relaciones múltiples no parece seguir una estructura jerárquica del grupo-2 al -4.

De acuerdo a estos resultados, podemos sacar varias conclusiones. En primer lugar, confirmamos la Hipótesis 2a: la distribución jerárquica se debe principalmente al número de relaciones en las que están implicados los miembros de los distintos grupos. Observamos esto en los valores de las medidas de densidad intra-grupos, que disminuyen sistemáticamente en todas las redes. Los valores disminuyen también en las relaciones inter-grupos, como nos lo muestran los valores totales de los porcentajes de las elecciones emitidas en el Cuadro IV.

En segundo lugar, confirmamos en parte la Hipótesis $\mathbf{2} \mathbf{b}$ : las relaciones múltiples caracterizan el centro de la red, pero su distribución no es jerárquica con respecto a los otros grupos. Con el fin de explorar este fenómeno, hemos representado las relaciones entre los miembros del grupo-5 en la Figura II. 


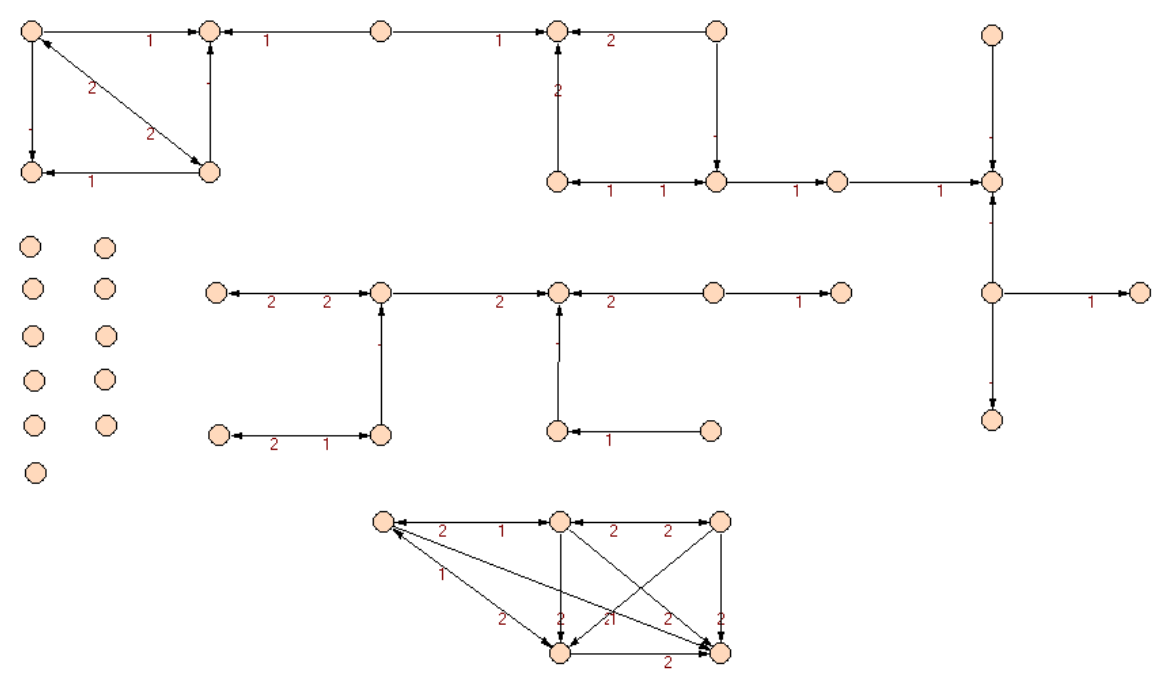

Figura II. Grafo de las relaciones entre los empresarios científicos miembros del Grupo-5 en la red de Consejo y Amistad.

Nota: El valor 1 de los arcos indica una relación simple de consejo o amistad, el valor 2 indica una relación múltiple de consejo y amistad.

Los miembros del grupo-5, que se encuentran más bien aislados del sistema de intercambio, mantienen a menudo relaciones múltiples (valor 2 de los arcos) y se concentran, algunos de ellos, en cliques densas. Este fenómeno, presente también en los otros grupos, no nos permite afirmar que la multiplicidad de los vínculos disminuye sistemáticamente a medida que los actores se alejan del centro de la red. Por el contrario, este resultado nos pone sobre la pista de un fenómeno de solidaridad específico que podría funcionar como una estrategia de compensación de la parte de los actores que, debido a su posición periférica, se verían perjudicados en la distribución de oportunidades.

En último lugar, en lo que se refiere a la proporción de relaciones recíprocas, el análisis conjunto de las dos redes muestra una distribución jerárquica, lo que confirma nuestra Hipótesis 2c. Sin embargo, el análisis independiente de las 2 redes (Cuadro V) revela una gran diferencia en la proporción de relaciones recíprocas en la red de Consejo al interior del grupo-1, donde está sobrerrepresentadas ( $43 \%$ ); y en los otros grupos, donde la reciprocidad es menos importante (13\%, $21 \%, 10 \%, 12 \%$ en el orden de aparición). En la red de Amistad, la proporción de relaciones recíprocas en el grupo-1 es menos importante (31\%) que en la red de Consejo. En cambio, el grupo- 2 muestra un escaso porcentaje de reciprocidad en la red de Consejo (13\%), mientras que más de un tercio de las relaciones de Amistad son recíprocas (36\%). De manera general, en los grupos - 2 a -5 , la reciprocidad es más importante en la red de Amistad que en la red de Consejo. 
Estos resultados nos llevan a preguntarnos sobre el rol que juegan las relaciones de Amistad para los actores que no ocupan las posiciones más centrales en el sistema de intercambio. Los recursos intercambiados en la red de Consejo se concentran en el grupo-1. En éste, las relaciones inter-organizacionales son muy densas y recíprocas, y sus miembros ( $15 \%$ de la población) atraen casi a la mitad de las elecciones relacionales (47\%, Cuadro IV) del conjunto de los actores. El status y la autoridad cognitiva (Lazega, 2001) de estos autores es reconocida por los otros actores. Sin embargo, las relaciones de amistad y las relaciones múltiples, que caracterizan también a los otros grupos, parecen mitigar en parte la supremacía del centro. Esto debido a que dichas relaciones permiten a los actores menos centrales mantener relaciones inter-organizacionales y romper así en parte con la distribución jerárquica que caracteriza la red de Consejo.

\subsection{Dos normas que orientan las elecciones relacionales}

Los análisis que llevamos a cabo con el fin de probar nuestra última serie de hipótesis ponen en relación dos características de las trayectorias profesionales de los empresarios con sus comportamientos relacionales.

\subsubsection{El rol de los fundadores en el sistema de intercambio}

La primera norma se define en torno a la condición de fundador o de no fundador del empresario. Nuestra investigación etnográfica nos permitió identificar un tipo de status social específico vinculado a la experiencia adquirida como fundador de una empresa en el ámbito de la Salud humana. Ser fundador significa haber sido el responsable de un proyecto científico y empresarial que debió recurrir al apoyo y la participación de numerosos y distintos tipos de actores. Ser fundador de una empresa que obtuvo los recursos para desarrollarse significa haber sido capaz de convencer del interés del proyecto a un gran número de interlocutores. En cambio, los dirigentes no fundadores se integran al equipo de dirección o sustituyen a los miembros fundadores una vez que la empresa ya encontró al menos los recursos básicos para desarrollarse. Su llegada a menudo coincide con la incorporación de un nuevo socio financiero o farmacéutico. Hemos explorado cómo esta distinción se cristaliza en el comportamiento relacional de los actores.

En la categoría de los fundadores hemos incluido a todos los dirigentes que declararon haber participado como fundador y accionista a la creación de una empresa en el sector de la Salud humana. A continuación, hemos observado las relaciones dentro y entre estos dos grupos. Los resultados se presentan en el cuadro VI. 


\begin{tabular}{|c|c|c|c|c|c|c|c|}
\hline & \multicolumn{2}{|c|}{ Outdegree Consejo } & \multirow[b]{2}{*}{$\begin{array}{c}\text { Total } \\
\text { indegree }\end{array}$} & \multicolumn{2}{|c|}{ Outdegree Amistad } & \multirow[b]{2}{*}{$\begin{array}{c}\text { Total } \\
\text { indegree }\end{array}$} \\
\hline & & Fundadores & $\begin{array}{c}\text { No } \\
\text { fundadores }\end{array}$ & & Fundadores & $\begin{array}{c}\text { No } \\
\text { fundadores }\end{array}$ & \\
\hline \multirow{3}{*}{$\begin{array}{c}\text { Fundadores } \\
\text { (116) }\end{array}$} & $\%$ todas las relaciones & $\mathbf{8 1 \%}$ & $\mathbf{8 1 \%}$ & 891 & $76 \%$ & $71 \%$ & 415 \\
\hline & $\%$ no recíprocas & $66 \%$ & $62 \%$ & 708 & $52 \%$ & $46 \%$ & 278 \\
\hline & \% recíprocas & $16 \%$ & $20 \%$ & 183 & $25 \%$ & $25 \%$ & 137 \\
\hline \multirow{4}{*}{$\begin{array}{c}\text { No } \\
\begin{array}{c}\text { Fundadores } \\
(48)\end{array} \\
\end{array}$} & $\%$ todas las relaciones & $19 \%$ & $19 \%$ & 204 & $24 \%$ & $29 \%$ & 137 \\
\hline & \% non recíprocas & $12 \%$ & $15 \%$ & 139 & $17 \%$ & $19 \%$ & 94 \\
\hline & \% recíprocas & $7 \%$ & $3 \%$ & 65 & $7 \%$ & $10 \%$ & 43 \\
\hline & Total outdegree & 810 & 285 & & 430 & 122 & \\
\hline \multirow{2}{*}{$\begin{array}{l}\text { Moyenne } \\
\text { Choix }\end{array}$} & outdegree & 8,1 & 6,1 & & 4,6 & 2,8 & \\
\hline & indegree & 8,8 & 4,4 & & 4,5 & 3,1 & \\
\hline
\end{tabular}

Cuadro VI. Relaciones intra- et inter- clases entre fundadores y no-fundadores y promedio de elecciones emitidas y recibidas en las redes de Consejo y Amistad a nivel inter-individual.

Nota: La cifra en las casillas en gris oscuro corresponde al total de las elecciones emitidas (outdegree) y recibidas (indegree) por las dos categorías de actores en las redes de Consejo y Amistad. De arriba abajo, las columnas dan cuenta del porcentaje de las elecciones emitidas (outdegree) por los actores fundadores y no fundadores en las dos redes analizadas separadamente, así como la proporción de los vínculos recíprocos y no recíprocos.

Por una parte, los fundadores solicitan consejo en promedio 8,1 veces, contra 6,1 los no fundadores; y declaran tener en promedio 4,6 amigos dentro de la población, contra 2,8 para los no fundadores (Cuadro VI). Los fundadores son también más elegidos, en particular en la red de Consejo donde son elegidos dos veces más en promedio que los no fundadores ( 8,8 contra 4,4 respectivamente). Estas medidas nos permiten confirmar nuestra Hipótesis 3a: los empresarios fundadores son más activos que los no fundadores en las redes de intercambio.

Por otra parte, las elecciones relacionales de los actores no se distribuyen de manera homogénea entre estas dos categorías. En la red de Consejo, más del 80\% de las elecciones emitidas por los fundadores y los no fundadores van dirigidas a los fundadores $(81 \%$ y $81 \%)$. Los no fundadores reciben solamente un $19 \%$ de las elecciones emitidas por las dos categorías. Un $20 \%$ de las elecciones emitidas por los no fundadores son recíprocos con los fundadores, mientras que solamente $3 \%$ de sus elecciones emitidas corresponden a relaciones recíprocas entre no fundadores. Esta cifra es tres veces superior en la red de Amistad (10\%). De hecho, en el sentido de nuestros análisis anteriores, el nivel de reciprocidad en la red de Amistad es más elevado en todas las configuraciones a excepción de las elecciones emitidas por los fundadores hacia los no fundadores que se mantienen al $7 \%$. En esta red, los fundadores son elegidos ligeramente menos que en la de Consejo, pero monopolizan de todas maneras más de dos tercios de las elecciones ( $76 \%$ y $71 \%$ para las dos categorías). Si se observan exclusivamente las relaciones recíprocas, más del $80 \%$ ( $20 \%$ de un total de $23 \%$ para esta configuración) de las relaciones recíprocas de los no fundadores son con fundadores, mientras que solamente $30 \%$ ( $7 \%$ de un total de $23 \%$ para esta configuración) de las relaciones recíprocas de estos últimos son con no fundadores. En lo que atañe las relaciones no recíprocas, solamente $15 \%$ de las relaciones de los fundadores van dirigidos a 
los no fundadores, mientras que más del $50 \%$ de las elecciones no correspondidas de los no fundadores van dirigidos a los fundadores.

Estos resultados muestran una preferencia en las elecciones de los actores en dirección de los fundadores. Su posición en el sistema de intercambio de recursos es especialmente importante en la red de Consejo. En esta red, observamos una proporción más elevada de las elecciones en dirección de los fundadores que en la red de Amistad. Confirmamos así nuestra Hipótesis 3b: el status social de los fundadores, medido a través de la proporción de las elecciones emitidas, es más importante que el de los no fundadores.

En lo que se refiere a los comportamientos, la mayor inversión relacional de los fundadores (H3a) está correlacionada con el alto status social que poseen (H3b). No se trata de una norma de homofilia, puesto que este comportamiento se observa también en los no fundadores. Se trata de una norma según la cual los empresarios orientan sus elecciones relacionales en dirección de aquellos que poseen la experiencia de crear una empresa en el sector de la Salud humana. Esta experiencia les confiere un status epistémico específico y los convierte en interlocutores preferenciales en el mecanismo de transmisión de conocimientos (representada por la red de Consejo) a la obra en esta industria.

\subsubsection{El rol de los científicos y financieros en el sistema de intercambio}

La segunda característica de los empresarios que hemos explorado se refiere al cargo desempeñado en el equipo de dirección ejecutivo de la empresa y a la manera en que ésta está asociada al intercambio informal de recursos a nivel interorganizacional.

La reconstitución de las relaciones informales de consejo a nivel interorganizacional en la industria del conocimiento exige un nivel de abstracción superior al necesario en los estudios intra-organizacionales. La diversidad y la especialización de los conocimientos ad hoc movilizados en este sector de actividad no permiten en general la rutinización de las actividades. En este contexto, el desafío para el investigador es encontrar, en el análisis de las actividades e interdependencias, los tipos de recursos intercambiados que corresponden al "máximo común denominador". Esto con el fin de mitigar en parte la especificidad de las actividades de las empresas y de los distintos cargos desempeñados en las empresas. El objetivo es poder observar a la vez el sistema de intercambio más extenso, más activo y también el más específico posible. Como hemos mencionado, los recursos más pertinentes que hemos podido definir tienen que ver con las actividades de gestión de los principales colaboradores de las empresas 
biotecnológicas, sin los cuales no podrían desarrollar sus proyectos. Todos los miembros del equipo dirigente se ven implicados en estas colaboraciones, independientemente del cargo que ocupen en la empresa. Las zonas de incertidumbre sobre las cuales se interrogó a los actores tienen que ver así con el entorno industrial en el que evolucionan. En este sentido, nuestra investigación se diseñó para que, al momento de dar cuenta de sus relaciones, los actores no estuvieran obligados a considerar el tipo de actividades específico de su cargo, sino más bien incitados a considerar las tareas como sub-proyectos en los cuales se ven implicados todos los miembros del equipo de dirección de la empresa. Dicho en otras palabras, les pedimos considerar "cada organización como un actor colectivo" (Lazega y al., 2007, p. 111), e indicarnos, entre los miembros de estas organizaciones, aquellos que les permitieron acceder a ciertos recursos.

Hemos clasificado los actores en 4 categorías: Director General (CEO), Director financiero (CFO), Director científico (CSO), y Director de operaciones (COO). Analizamos las relaciones al interior y entre estas 4 clases. Los resultados presentados en el cuadro VII se refieren a las relaciones de Consejo y Amistad observadas conjuntamente. La primera parte del cuadro presenta los resultados del análisis de la Independencia estadística en las relaciones entre los miembros de las 4 clases. Este método permite tomar en cuenta los comportamientos relacionales consustanciales a cada grupo en la medida en que la importancia relativa de las elecciones emitidas y recibidas no se calcula en función del número de las elecciones posibles, sino en función del número de elecciones observadas para cada tipo de actor. La segunda parte del cuadro presenta la proporción de elecciones emitidas por los actores en dirección de los miembros de los otros grupos.

\begin{tabular}{|c|c|c|c|c|c|c|}
\hline \multicolumn{7}{|c|}{ Relaciones de preferencia (= Relaciones observadas/Valores de independencia estadística } \\
\hline & CEO (88) & CFO (20) & CSO (23) & \multicolumn{3}{|c|}{$\mathrm{COO}(33)$} \\
\hline CEO & 1,00 & 0,93 & 1,04 & \multicolumn{3}{|l|}{1,02} \\
\hline CFO & 1,01 & 1,61 & 0,39 & \multicolumn{3}{|l|}{0,93} \\
\hline CSO & 1,02 & 0,57 & 1,35 & \multirow{2}{*}{$\begin{array}{l}0,96 \\
0,99\end{array}$} & & \\
\hline $\mathrm{COO}$ & 0,98 & 1,01 & 1,11 & & \multicolumn{2}{|c|}{ Outdegree } \\
\hline \multicolumn{5}{|c|}{ Porcentajes de las elecciones emitidas intra- et inter-grupos } & Total & Promedio \\
\hline CEO & $64 \%$ & $10 \%$ & $11 \%$ & $16 \%$ & 825 & 6,00 \\
\hline CFO & $65 \%$ & $17 \%$ & $4 \%$ & $14 \%$ & 176 & 4,05 \\
\hline CSO & $66 \%$ & $6 \%$ & $14 \%$ & $15 \%$ & 116 & 3,78 \\
\hline $\mathrm{COO}$ & $63 \%$ & $11 \%$ & $11 \%$ & $15 \%$ & 336 & 3,91 \\
\hline Indegree & 929 & 154 & 148 & 222 & & \\
\hline
\end{tabular}

Cuadro VII. Relaciones intra- et inter- clases entre les empresarios por tipo de cargo desempeñado en la empresa.

Nota: la primera parte del cuadro presenta los resultados del análisis de la Independencia estadística. Las relaciones de preferencia corresponden al cociente de las relaciones observadas y los valores no preferenciales calculados a partir de los comportamientos relacionales observados. La segunda parte 
presenta la proporción de las elecciones emitidas por los actores (eje vertical) en dirección de los miembros de cada grupo (eje horizontal).

En la primera parte del Cuadro VII, las columnas y las líneas de los CEO y COO se caracterizan por medidas bastante homogéneas en torno a 1. Esto significa que las elecciones emitidas y recibidas por estos actores son equivalentes a los valores no preferenciales construidos a partir de los comportamientos relacionales observados. En cambio, los valores de las relaciones de los CFO y CSO muestran preferencias muy acentuadas. Por una parte, las relaciones entre estos dos grupos presentan preferencias negativas (0,39 y 0,57 respectivamente), lo que indica que estas relaciones inter-clases están infrarrepresentadas. Por otra parte, los dos grupos eligen al interior de sus grupos a más interlocutores de lo que lo harían si no existiese ninguna preferencia (1,61 y 1,35 respectivamente), lo que indica que los vínculos intra-clase están sobrerrepresentados.

La segunda parte del cuadro muestra la distribución de las elecciones intra- e interclases. Observamos que los CEO atraen casi dos tercios de las elecciones emitidas. Estos resultados ponen de manifiesto también que solamente $4 \%$ de las elecciones emitidas por los CFO van dirigidos a los CSO, y que, en el sentido opuesto, solamente $6 \%$ de las elecciones emitidas por los CSO van dirigidos a los CFO.

La Figura III nos permite visualizar estos resultados. En ella representamos a los miembros de los dos grupos y sus relaciones de consejo y amistad. Borramos las relaciones entre los miembros de una misma empresa para tener en cuenta exclusivamente las relaciones inter-organizacionales. 


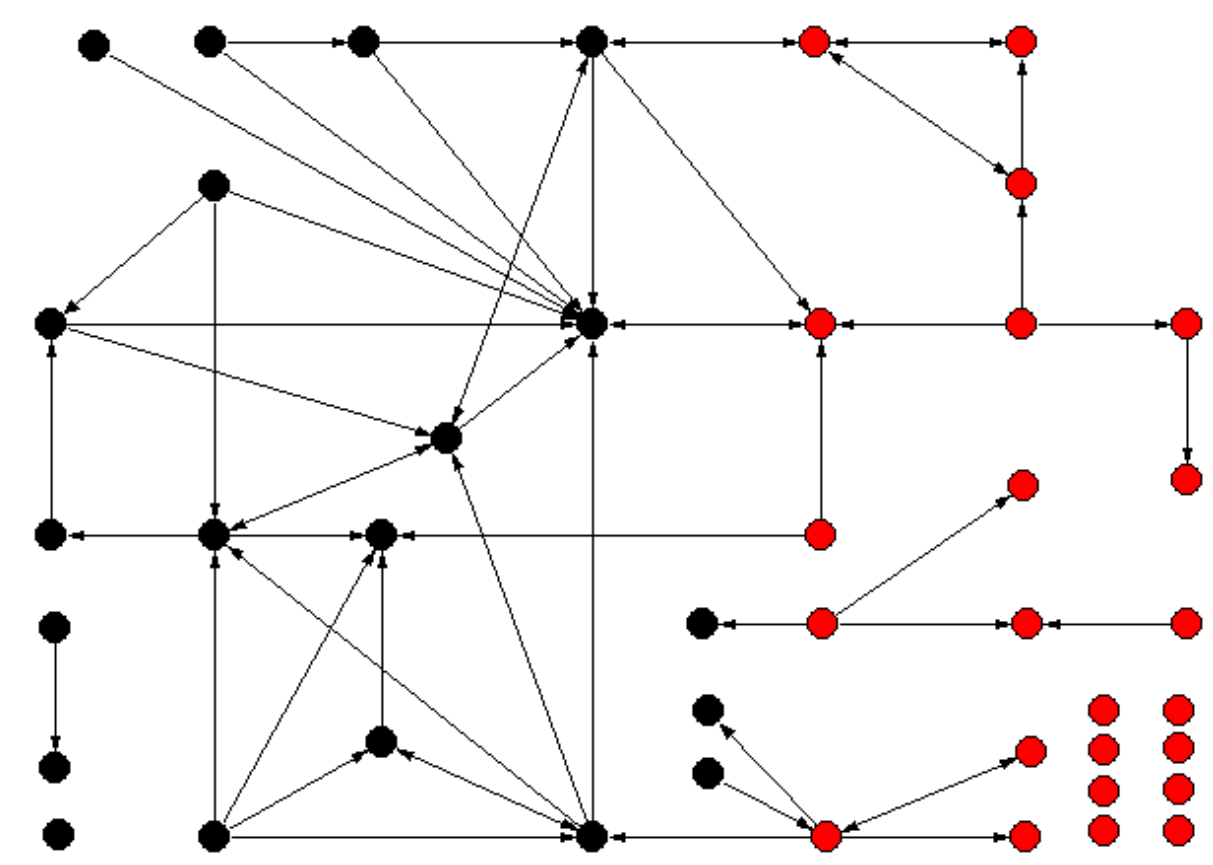

Figura III. Grafo de las relaciones inter-organizacionales de Consejo y Amistad entre los CFO (20) y los CSO (23)

Nota: los nodos en negro representan a los CFO, los nodos en rojo a los CSO. Los CFO mantienen más relaciones que los CSO.

Los CFO (en negro) mantienen más relaciones que los CSO (en rojo). En los primeros, solamente 4 actores están aislados (contamos a los 3 actores que mantienen solamente relaciones con CSO). En el grupo de los CSO, 8 actores están aislados. Solamente 6 de 56 relaciones son inter-clases, de los cuales 2 son recíprocas.

Estos resultados nos permiten confirmar nuestra Hipótesis 3c: independientemente del nivel de actividad (elecciones emitidas) y el nivel de status (elecciones recibidas) de los actores por cargo en la empresa, constatamos una preferencia negativa y mutua entre los responsables científicos y los responsables financieros.

El estudio etnográfico que realizamos nos hace pensar que esta ausencia de relaciones está en parte vinculada al encuentro, a menudo violento, entre el mundo de la IA y el de la finanza. Las distintas lógicas de valorización del mundo científico y del sector privado (Saives y al. 2005, Noisi, 2003), las dificultades temporales vinculadas a la duración de los fondos de inversión (Pisano, 1990), así como las dificultades terapéuticas y reglamentarias del desarrollo de los productos constituyen un conjunto de obstáculos que requieren la coordinación de los dos "sistemas de pensamiento" (systèmes de pensé). Una empresa de biotecnología 
representa el duro trabajo de adaptación mutua entre estos mundos. Este ajuste requiere que los actores negocien su identidad (Lazega, 1992), poniendo así en peligro la manera en la que han construido su vida profesional. El científico y financiero representan el uno para el otro este desafío, que se refleja en su comportamiento relacional por una norma de preferencia negativa entre ellos.

Nuestros resultados muestran también que dicha preferencia negativa que caracteriza las relaciones entre los CSO y los CFO se acompaña de una norma de homofilia al interior de cada categoría. La Figura III pone de manifiesto que estos dos tipos de actores tienden a distribuir sus elecciones entre los miembros de su misma categoría. La ausencia de relaciones entre estos actores y las otras categorías (CEO y COO, ver Cuadro VII), nos permite pensar que estas dos normas se refuerzan mutuamente. Éstas serían la expresión de una separación en el sistema de intercambio de recursos (para un análisis detallado del movimiento de polarización que caracteriza los intercambios en este medio ver Pina-Stranger y Lazega, 2010). Por una parte, gracias a la norma de homofilia asociada al cargo en la empresa de los CSO y los CFO, estos actores acceden más fácilmente a los recursos que circulan dentro de su grupo, beneficiándose de y participando en, un proceso de solidaridad específico. En cambio, la norma de preferencia negativa inter-clases, reduce la posibilidad de verse beneficiados por los recursos que circulan en la categoría opuesta, lo que da cuenta de un proceso de exclusión recíproco entre CSO y CFO. Esta dinámica parece beneficiar más a los CFO que pueden sacar provecho de un sistema de intercambio más cohesivo que el de los CSO, como nos lo muestra la Figura III, y las medidas de las relaciones intra-clase de los CFO (Cuadro VII). Por último, vale la pena señalar que los CSO que mantienen relaciones con CFO son los más centrales de su clase, mientras que en la clase de los CFO esto es menos cierto.

\section{Conclusiones}

En el marco de los estudios sobre la cooperación entre competidores, hemos presentado el enfoque inter-individual como un complemento necesario del enfoque contractual generalmente utilizado en el estudio de la industria biotecnológica. El examen de las relaciones personales que mantienen los dirigentes de las empresas biotecnológicas nos ha permitido formular hipótesis relativas al modo de funcionamiento del sistema de intercambio a la obra en este medio. Hemos podido así superar la descripción puramente económica de las relaciones interorganizacionales, para explorar las formas y las normas sociales que caracterizan esta industria. 
Por una parte, establecimos la existencia de un sistema de intercambio general, caracterizado por la circulación de distintos tipos de recursos. La forma de esta estructura social no es aleatoria ya que hemos podido identificar una distribución jerárquica de los recursos, donde el centro se diferencia de la periferia por la existencia de relaciones más densas, múltiples y recíprocas. Las relaciones de consejo, que dan cuenta de áreas específicas de experticia vinculadas a la gestión de los principales socios de las empresas biotecnológicas, se concentran en el centro, mientras que la amistad y las relaciones múltiples se distribuyen de una manera más homogénea en los distintos grupos periféricos. Nuestro estudio sugiere que este fenómeno pueda indicar la existencia de un mecanismo de compensación que permite a los actores periféricos participar al sistema de intercambio interrelacional, incluso si su posición social, y, en particular, su status epistémico (representado por las elecciones recibidas en la red de Consejo) es menos favorables.

Por otra parte, se descubrieron dos normas que caracterizan el intercambio de recursos. La primera se refiere al rol de los fundadores en el sistema de intercambio informal. Existe una tendencia al interior del grupo de empresarios que estudiamos a orientar sus preferencias relacionales en dirección de aquellos que ya han tenido la experiencia de crear una empresa en el sector de la Salud humana. Esta experiencia les confiere un status epistémico específico, que se traduce en un rol preponderante en la transmisión informal de conocimientos en esta industria.

La segunda norma concierne la manera en que los distintos cargos en la empresa determinan el intercambio informal de recursos a nivel inter-organizacional. En particular, hemos mostrado que existe una preferencia negativa que caracteriza las relaciones entre los Directores científicos (CSO) y los Directores financieros (CFO). Esta tendencia se acompaña de una norma de homofilia al interior de estas categorías. Este resultado sugiere la existencia de un mecanismo doble de solidaridad y de exclusión que puede a la vez favorecer y dañar la eficiencia del sistema de intercambio informal en esta industria.

Las normas de intercambio que hemos identificado, gracias al estudio de los comportamientos relacionales, reflejan en parte la manera en que algunas dificultades vinculadas a la estructuración global de esta industria determinan las formas de intercambio a la obra en este medio. Nos parece que el status social más importante de los fundadores tiene relación con los innumerables obstáculos, reales o supuestos, que estos han debido superar. 
Como indica Freeman (1991), en el estudio de los procesos de innovación, la visión del empresario como un héroe excepcional capaz de imponer su voluntad creativa domina el pensamiento económico desde Schumpeter. En este contexto, el creador de empresa es considerado como el más eminente representante de los empresarios. Esto es particularmente cierto en la industria biotecnológica, en la que a las dificultades "corrientes" se debe agregar aquellas vinculadas al desarrollo de los productos terapéuticos. Esta creencia, más o menos justificada, puede ser la causa de las tendencias relacionales observadas.

Por su parte, el fenómeno de exclusión mutua entre los CSO y los CFO, es el reflejo quizá del difícil proceso de ajuste por el que debe pasar un descubrimiento científico para convertirse en una invención terapéutica. Esto iría en el sentido de las proposiciones hechas por Owen-Smith y al. (2002) para quien el sector del IA en Europa se integra poco al de la investigación clínica.

\section{Bibliografía}

Aharonsona, B., Baum, J., Plunket, A. (2008) "Inventive and uninventive clusters: The case of Canadian biotechnology", Research Policy (37) 1108-1131.

Audretsch, D., Stephan, P. (1996) « Company-Scientist Locationai Links: The Case of Biotechnoiogy », The American Economic Review, 641-652.

Autant-Bernard, C., Mangematin, V., Massard, N. (2006) "Creation of Biotech in France", Small Business Economics (26) 173-87.

Batagelj, V., Mrvar, A. (2008) Pajek, Program for Analysis and Visualization of Large Networks, Reference Manual, List of commands with short explanation, version 1.22, Ljubljana.

Baum, J., Calabrese, T., Silverman, B. (2000) "Don't go it alone: Allieance network composition and startups' performance in Canadian biotechnology", Strategic Management J ournal (21) 267-94.

Blau, P. (1964) Exchange and Power in social life. Transaction Publishers, New Brunswick.

Borgatti, S.P., Everett, M.G. and Freeman, L.C. (2002) Ucinet for Windows: Software for Social Network Analysis. Harvard, MA: Analytic Technologies.

Boufaden, N., Plunket, A. (2005) "Investigating technological and geographic proximity on forms' innovation in an immature cluster: the Paris area biotech cluster", DRUID Conference, Copenhagen.

Casper, S. (2007) "How do technology clusters emerge and become sustainable? Social network formation and inter-firm mobility within the San Diego biotechnology cluster", Research Policy (36) 438-455.

Champenois, Claire, (2008) «La co-localisation d'entreprises innovantes comme non choix. L'exemple de l'industrie allemande des biotechnologies », Géogaphie Economie Entreprise (10) 61-86.

Chen, R., Shyu, J., Tzeng, G. (2006) « The Policy of High-Tech Industry Development: The Case of Location Assessment for Biotech Industry Parks in Taiwan «, Review of Policy Research (23) 580-606. 
Coenen, L., Moodysson, J., Asheim, B. (2004) “Nodes, Networks and Proximities: On the Knowledge Dynannics of the Medicon Valley Biotech Cluster", European Planning Studies (12) 1003-1018.

Filiou, D. (2005) “Exploration and exploitation in inter-organizational learning: evidence from biotechnology sector in the UK between 1991 and 2001", DRUID Tenth Anniversary Summer Conference Copenhagen, Denmark, June 27-29, 2005.

Freeman, C. (1991) "Networks of innovators: A synthesis of research issues", Research Policy (20) 499-514.

Gertler, M. S., Levitte, Y. M. (2005) «Local Nodes in Global Networks: The Geography of Knowledge Flows in Biotechnology Innovation », Industry and Innovation (12) 487-507.

Gilding, M. (2008) “'The tyranny of distance': Biotechnology networks and Clusters in the antipodes", Research Policy (37) 1132-1144.

Jaffe, A., Trajtenberg, M., Henderson, R., (1993) “Geographic localization of knowledge spillovers as evidence by patent citations", Quarterly Journal of Economics (63) 577-98.

Ingram, P., Roberts, P. (2000) "Friendships among Competitors in the Sydney Hotel Industry", American Sociological Review (106) 387-423.

Lazega, E. (1992), The Micropolitics of Knowledge: Communication and Indirect Control in Workgroups. A. de Gruyter, New York.

(2001), The Collegial Phenomenon: The Social Mechanisms of Cooperation Among Peers in a Corporate Law Partnership. Oxford University Press, Oxford.

(2003) «Rationalité, discipline sociale et structure», Revue Française de S̄ociologie (44) 305-29.

_ (2005) "Capital social, processus sociaux et capacité d'action collective", dans Le capital social - Performance, équité et réciprocité, Bevort, A. et MICHEL, E. (eds), La découverte, Paris.

(2008). Théorie de la coopération entre concurrents. In P. Steiner \& F. Vatin Traîté de sociologie économique. Paris: PUF, Quadrige, pp. 1-22.

Lazega, E., Jourda, M.T., Mounier, L., Stoffer, R. (2007), « Des poissons et des mares : I'analyse de réseaux multiniveaux », Revue Française de Sociologie (48) 93-131.

Lazega, E. et Mounier, L. (2002) "Interdependent entrepreneurs and the social discipline of their cooperation: The research program of structural economic sociology for a society of organizations », in Olivier Favereau et Emmanuel Lazega (eds)", Conventions and Structures in Economic Organization: M, Cheltenham, Edward Elgar Publishing, pages 147-199.

Lemarié, S., Mangematin, V., Torre, A. (2001) "Is the creation and development of biotech localized? Conclusion drawn from the French case", Small Business Economics (17) 61-76.

Luukkonen, T. (2005) "Variability in organizational forms of biotechnology firms", Research Policy (34) 555-70.

Mangematin,V., Lemarie, S., Boissin, J.-P., Catherine, D., Corolleur, F., Corolini, R., Trommetter, M.( 2003) "Development of SMEs and heterogeneity of trajectories: the case of biotechnology in France", Research Policy (32) 621-638.

Niosi, J. (2003) «Alliances are not enough explaining rapid growth in biotechnology firms », Research Policy (32) 737-750. 
Nooy, W. de, Mrvar, A., Batagelj, V. (2005), Exploratory Social Network Analysis with Pajek, New York, Cambridge University Press.

Orsenigo, L., Pammolli, F., Riccaboni, M. (2000) "Technological change and network dynamics: lessons from the pharmaceutical industry", Research Policy (30) 485508.

Owen-Smith, J., Riccaboni, M., Pammolli, F., Powell, W.W. (2002) "A Comparison of U.S. and European University-Industry Relations in the Life Sciences", Management Science (48) 24-43.

Owen-Smith, J., Powell, W.W. (2004) "Knowledge networks as channels and conduits: The effects of spillovers in the Boston biotechnology community", Organization Science (15) 5-21.

Pina-Stranger, A. (2009). Transfert technologique et processus collectif d'apprentissage dans l'industrie des biotechnologies en France. Sociologie Santé, 30 (Medicaments et société: entre automédication et dépendance), 27-49.

Pina-Stranger, A., Lazega, E. (en prensa, 2010). Inter-organizational collective learning: the case of biotechnology in France. European journal of international management.

Pisano, G. (1990) "The R\&D boundaries of the firm: an empirical analysis - research and development - Technology, Organizations, and Innovation", Administrative Science Quarterly (35) 153-176.

Pisano, G. (1991) "The governance of innovation: vertical integration and collaborative arrangements in the biotechnology industry", Research Policy 20: 237-249.

Powell, W.W. (1990) "Neither Market nor Hierarchy: Network Forms of Organization", Research in Organizational Behavior (12) 295-336.

(1996) "Inter-Organizational Collaboration in the Biotechnology Industry", Journal of Institutional and Theoretical Economics.

(1998), "Learning from collaboration: knowledge and networks in the biotechnology and pharmaceutical industries", California Management Review.

Powell, W. W., Koput, K. W., Smith-Doerr (1996), "Inter-Organizational Collaboration and the locus of innovation: networks of learning in the biotechnology", Administrative Science Quarterly, (41) 116-45.

Powell, W. W., Koput, K. W., Smith-Doerr, L. and Owen-Smith, J. (1999), "Network position and firm performance: Organizational returns to collaboration in the biotechnology industry," Research in the Sociology of Organizations (16) 129-159.

Powell, W. W., Koput, K. W., Bowie, J., Smith-Doerr, L. (2002) “The Spatial Clustering of Science and Capital: accounting for Biotech Firm-Venture Capital relationships", Regional Studies (36) 291-305.

Reynaud, J.D. (1989) Les Règles du jeu : L'action collective et la régulation sociale. Armand Colin, Paris.

Saives, A. L., Ebrahimi, M., Desmarteau, R., Garnier, C. (2005) « Les logiques d'évolution des entreprises de biotechnologie », RFG (155) 153-171.

Salman, N., Saives, A. L. (2005) “Indirect networks: an intangible resource for biotechnology innovation", R\&D Management (35) 203-215.

Senker, J., Sharp, M. (1997) “Organizational learning in cooperative alliances: Some case studies in Biotechnology", Technology Analysis \& Strategic Management (9) 35-51. 
Uzzi, B. (1997) "Social Structure and Competition in Interfirm Networks: The Paradox of Embeddedness", Administrative Science Quarterly (42) 35-67.

Wasserman, S., Faust, K. (1994) Social Network Analysis: Methods and Applications. Cambridge University Press, New York.

White, H.C. (1981) "Where Do Markets Come From?", The American Journal of Sociology (87) 517-547.

Zucker, L.G., Darby, M.R., Armstrong, J. (1998) "Geographically localized knowledge: spillovers or markets?" Economic Inquiry, (36) 65-86.

Zucker, L.G., Darby, R. (2001) “Capturing Technological Opportunity Via Japan's Star Scientists: Evidence from Japanese Firms' Biotech Patents", Journal of Technology Transfer (26) 37-58. 